\title{
Quantum scrambling with classical shadows
}

\author{
Roy J. Garcia $\odot,^{1, *}, \dagger$ You Zhou ${ }^{\circ},{ }^{1,2, *},+$ and Arthur Jaffe ${ }^{10}{ }^{1, \S}$ \\ ${ }^{1}$ Department of Physics, Harvard University, Cambridge, Massachusetts 02138, USA \\ ${ }^{2}$ School of Physical and Mathematical Sciences, Nanyang Technological University, 637371, Singapore
}

(Received 16 March 2021; accepted 21 June 2021; published 13 August 2021)

\begin{abstract}
Quantum dynamics is of fundamental interest and has implications in quantum information processing. The four-point out-of-time-ordered correlator (OTOC) is traditionally used to quantify quantum information scrambling under many-body dynamics. Due to the OTOC's unusual time ordering, its measurement is challenging. We propose higher-point OTOCs to reveal early-time scrambling behavior, and present protocols to measure any higher-point OTOC using the shadow estimation method. The protocols circumvent the need for time-reversal evolution and ancillary control. They can be implemented in near-term quantum devices with single-qubit readout.
\end{abstract}

DOI: 10.1103/PhysRevResearch.3.033155

\section{INTRODUCTION}

Quantum scrambling describes the delocalization of quantum information in quantum chaotic systems [1,2]. Much of the interest in scrambling derives from the study of black holes, the fastest scramblers in nature [2-5]. The holographic duality permits the investigation of black hole scrambling via the probing of certain models in condensed matter physics, like the Sachdev-Ye-Kitaev model [6,7]. Scrambling can be studied by probing the four-point out-of-time-ordered correlator (OTOC) [8-10]. This correlator can be used to quantify chaos in many-body systems ranging from a non-integrable Ising model [11] to the Dicke model [12-14]. For fast scrambling systems $[6,7,15,16]$, this correlator decays exponentially within the scrambling time, according to a Lyapunov exponent. Measuring OTOCs in this regime can be used to investigate models with holographic duals. OTOCs can also be used to characterize many-body localized systems [17-22].

The time ordering of the OTOC makes its measurement tedious. Nevertheless, there are experimental protocols to measure the four-point OTOC. Protocols based on timereversal evolution have been demonstrated [23,24]. The OTOC has also been measured using a nuclear magnetic resonance quantum simulator [25] and a two-dimensional system of 53 superconducting qubits [26]. Recent investigations of scrambling have searched for measurement protocols that circumvent the need for time reversal. To distinguish between

\footnotetext{
*These authors contributed equally to this work.

†roygarcia@g.harvard.edu

‡zyqphy@gmail.com

§arthur_jaffe@ @arvard.edu
}

Published by the American Physical Society under the terms of the Creative Commons Attribution 4.0 International license. Further distribution of this work must maintain attribution to the author(s) and the published article's title, journal citation, and DOI. scrambling and decoherence, a teleportation protocol that measures the OTOC in the large-time limit was developed in Ref. [27] and demonstrated in [28,29]. A method based on statistical correlations computes the four-point OTOC in terms of experimentally friendly correlators [30]; it was demonstrated in Ref. [31]. Although quantum chaos is often studied through the four-point OTOC, it is suspected that scrambling is sensitive to higher-point correlators [28,32,33]. Thus, one desires a protocol to measure higher-point OTOCs, especially one without time reversal or ancillary control operations.

We present protocols to measure any higher-point outof-time-ordered correlator using classical shadows [34,35], which is an efficient scheme recently proposed to predict functions of quantum states. While nonlinear functions are often computed by preparing multiple copies of a state [36-40], classical shadows allow us to bypass this preparation by measuring a single copy of the state. Our protocols avoid time reversal and can probe OTOCs at any time. While ancillary qubits are integrated, we do not require that they exert an interaction on the system [41]. We find that the eight-point OTOC reveals early-time information delocalization not present in the four-point OTOC, making it a promising candidate to probe scrambling dynamics.

We provide a statistical error analysis to show that our protocols are more efficient than brute force tomography. Since our protocols express OTOCs in terms of nonlinear functions of a state, we give a refined variance analysis on nonlinear functions which rely on the product structure of the target state (i.e., $\rho^{\otimes k}$ ), establishing a tighter bound than previous works $[35,42]$; a result which is of independent interest. We also numerically simulate our protocols in a nonintegrable, mixedfield Ising model and show that they can be implemented by current experimental platforms containing a moderate number of qubits.

\section{A. Higher-point correlators}

Scrambling is often quantified through operator spreading, described as follows. Consider a quantum many-body system 
consisting of $N$ qubits that evolves by a chaotic Hamiltonian, $H$. Let $W$ and $V$ be local, unitary operators on Hilbert space $\mathcal{H}_{d}$ of dimension $d=2^{N}$. "Local" refers to unitaries which act on different qubits. Assume $W$ and $V$ commute at time $t=0$. The Heisenberg operator

$$
W(t) \equiv U_{H}^{\dagger}(t) W U_{H}(t)
$$

evolves with $U_{H}(t)=e^{-i H t}(\hbar=1)$. The quantum butterfly effect $[5,43]$ states that $[W(t), V]$ grows as $W(t)$ delocalizes, i.e., spreads throughout the system. Intuitively, $W(t)$ eventually acts nontrivially at the location of $V$, at which point the commutator no longer vanishes. The size of the commutator can therefore be used to quantify the spreading of $W(t)$ and hence measure scrambling.

To measure the growth of the commutator, it is common to take its norm [44]. The Hilbert-Schmidt norm is most often employed $[9,10,17]$ :

$$
\|[W(t), V]\|_{\mathrm{HS}}=\left[\operatorname{Tr}\left\{|[W(t), V]|^{2}\right\}\right]^{1 / 2} .
$$

To measure this quantity, we interpret the trace as (up to a normalization) the expectation value of $|[W(t), V]|^{2}$ over the infinite temperature thermal state $\rho_{\infty}=\frac{1}{d} I_{N}$. By adopting the notation $\langle\cdot\rangle \equiv \operatorname{Tr}\left\{\rho_{\infty} \cdot\right\}$, the expectation value is

$$
\left\langle|[W(t), V]|^{2}\right\rangle=2\left(1-\operatorname{Re}\left\{C_{4}(t)\right\}\right),
$$

where the four-point out-of-time-ordered correlator is defined as

$$
C_{4}(t)=\left\langle W^{\dagger}(t) V^{\dagger} W(t) V\right\rangle .
$$

Scrambling causes this correlator to decay to near zero. Although $C_{4}(t)$ has been analyzed extensively in the literature, it does not describe the complete evolution of the commutator. Higher-point correlators are necessary to reveal new, earlytime scrambling.

To extract higher-point correlators, we measure the commutator growth using the Schatten $2 n$-norm for positive integer $n$,

$$
\|[W(t), V]\|_{2 n}=\left[\operatorname{Tr}\left\{|[W(t), V]|^{2 n}\right\}\right]^{1 / 2 n} .
$$

This is computed by measuring the expectation value $\left\langle|[W(t), V]|^{2 n}\right\rangle=\frac{1}{d} \operatorname{Tr}\left\{|[W(t), V]|^{2 n}\right\}$. By expanding out $|[W(t), V]|^{2 n}$, the Schatten $2 n$-norm can be expressed in terms of a linear combination of higher-point correlators

$$
\|[W(t), V]\|_{2 n}=\left[d \sum_{k=0}^{n} b_{k}(n) \operatorname{Re}\left\{C_{4 k}(t)\right\}\right]^{1 / 2 n},
$$

for some coefficients $b_{k}(n)$. The $4 k$-point OTOC, for a nonnegative integer $k$, is defined as

$$
C_{4 k}(t) \equiv\left\langle\left(W^{\dagger}(t) V^{\dagger} W(t) V\right)^{k}\right\rangle .
$$

We propose a physical interpretation of these correlators in Appendix A. A form of this kind of higher-point OTOC has also been studied in $[33,45,46]$.

In this work, we develop measurement protocols to estimate $C_{4 k}(t)$ by using randomized measurements $[47,48]$ via the classical shadow framework [35]. The applications of randomized measurements range from quantum many-body physics, such as the detection of topological order $[49,50]$ and entanglement entropy [51,52], to quantum information and quantum foundations, such as the extraction of entanglement negativity [42,53], quantum benchmarking [54,55], and entanglement detection without reference frames [56-60].

The paper is organized as follows. In Sec. II, we adopt a global random ensemble to measure the eight-point correlator $C_{8}(t)$, which reveals scrambling earlier than $C_{4}(t)$. In Sec. III, we propose three complementary protocols based on shadow tomography, only requiring random Pauli measurements on individual qubits. We give analytical variance upper bounds for a sample protocol in Sec. IV and show the protocol is more efficient than direct tomography. In Sec. V we present numerical simulations for the predicted and estimated OTOC values. We give conclusions and provide an outlook in Sec. VI.

\section{GLOBAL PROTOCOL FOR EIGHT-POINT OTOC}

We motivate our search for a novel protocol to measure higher-point correlators by first extending the four-point OTOC protocol developed in [30] to evaluate $C_{8}(t)$. The protocol implements global random unitaries and demonstrates new scrambling dynamics in $C_{8}(t)$.

Assume $W$ and $V$ are unitary, traceless, and Hermitian operators on $\mathcal{H}_{d}$. Defining operators $A_{1}=W(t)$ and $A_{2}=$ $V W(t) V$, the four-point and eight-point OTOCs are

$$
C_{4}(t)=\left\langle A_{1} A_{2}\right\rangle, \quad C_{8}(t)=\left\langle A_{1} A_{2} A_{1} A_{2}\right\rangle .
$$

$A_{1}$ and $A_{2}$ are also unitary, traceless, and Hermitian operators. Let $U$ be a unitary on $\mathcal{H}_{d}$ randomly sampled from the Haar measure on the unitary group. Define the notation for the integral over the Haar measure as $\overline{(\cdots)}=\int_{\text {Haar }} d U(\cdots)$. Define the expectation value over the pure state $\rho_{0} \in \mathcal{H}_{d}$ as $\langle\cdot\rangle_{\rho_{0}}=\operatorname{Tr}\left\{\rho_{0} \cdot\right\}$. One can prove that (see Appendix D)

$$
\begin{aligned}
\left\langle A_{1} A_{2}\right\rangle=(d & +1) \overline{\left\langle U^{\dagger} A_{1} U\right\rangle_{\rho_{0}}\left\langle U^{\dagger} A_{2} U\right\rangle_{\rho_{0}}}, \\
\left\langle A_{1} A_{2} A_{1} A_{2}\right\rangle= & \frac{1}{2}(d+1)(d+2)(d+3) \\
& \times \overline{\left\langle U^{\dagger} A_{1} U\right\rangle_{\rho_{0}}^{2}\left\langle U^{\dagger} A_{2} U\right\rangle_{\rho_{0}}^{2}} \\
& -d\left\langle A_{1} A_{2}\right\rangle^{2}-\frac{1}{2}(d+4),
\end{aligned}
$$

for any pure state $\rho_{0}$, even, for example, $|0\rangle\left\langle\left. 0\right|^{\otimes N}\right.$. In terms of $W(t)$ and $V$, the OTOCs are

$$
\begin{aligned}
C_{4}(t)=(d+1) \overline{\left\langle U^{\dagger} W(t) U\right\rangle_{\rho_{0}}\left\langle U^{\dagger} V^{\dagger} W(t) V U\right\rangle_{\rho_{0}}} & \\
C_{8}(t)= & \frac{1}{2}(d+1)(d+2)(d+3) \\
& \times \overline{\left\langle U^{\dagger} W(t) U\right\rangle_{\rho_{0}}^{2}\left\langle U^{\dagger} V^{\dagger} W(t) V U\right\rangle_{\rho_{0}}^{2}} \\
& -d C_{4}^{2}(t)-\frac{1}{2}(d+4) .
\end{aligned}
$$

Equation (12) reveals that $C_{8}(t)$ contains dynamics not captured by $C_{4}(t)$. Its third term is constant and does not affect the dynamics. The second term depends only on $C_{4}(t)$. The "hidden" dynamics arise due to the new term $\overline{\left\langle U^{\dagger} W(t) U\right\rangle_{\rho_{0}}^{2}\left\langle U^{\dagger} V^{\dagger} W(t) V U\right\rangle_{\rho_{0}}^{2}}$.

To evaluate the OTOCs, we measure $\left\langle U^{\dagger} W(t) U\right\rangle_{\rho_{0}}$ and $\left\langle U^{\dagger} V^{\dagger} W(t) V U\right\rangle_{\rho_{0}}$ with global random unitaries as follows. 
(1) Randomly sample a unitary $U$ from the Haar measure on the unitary group.

(2) Prepare pure state $\rho_{0}$ and evolve with $U$. Then evolve with $U_{H}(t)$. Measure $W$.

(3) Repeat step 2 many times to compute the expectation value $\left\langle U^{\dagger} W(t) U\right\rangle_{\rho_{0}}$.

(4) Prepare $\rho_{0}$ and evolve with $U$. Apply $V$, then evolve with $U_{H}(t)$. Measure $W$.

(5) Repeat step 4 many times to compute the expectation value $\left\langle U^{\dagger} V^{\dagger} W(t) V U\right\rangle_{\rho_{0}}$.

(6) Repeat steps 1-5 with many random unitaries.

We compute $\overline{\left\langle U^{\dagger} W(t) U\right\rangle_{\rho_{0}}^{2}\left\langle U^{\dagger} V^{\dagger} W(t) V U\right\rangle_{\rho_{0}}^{2}}$ by calculating $\left\langle U^{\dagger} W(t) U\right\rangle_{\rho_{0}}^{2}\left\langle U^{\dagger} V^{\dagger} W(t) V U\right\rangle_{\rho_{0}}^{2}$ for each $U$, then averaging.

Although a subensemble can be substituted in for the Haar measure on the unitary group via unitary $t$-design $[33,61]$, an ensemble forming a 4-design is needed to measure $C_{8}(t)$. Note that the Clifford group forms a 3-design [62-64], but not a 4-design [63]. One can generate an approximate $t$-design through a random local circuit [65], in particular, by inserting few $T$ gates in the Clifford circuits [66]. That is, one must apply a more random (chaotic) unitary ensemble to access higher-point scrambling features. Since generating global random unitaries is experimentally challenging, Ref. [30] adapts its global protocol to local unitaries, greatly simplifying the measurement procedure. However, higher-point correlators are difficult to evaluate using this local protocol, due to the lack of degrees of freedom needed to construct larger permutation operators. This kind of no-go result is also observed in the measurement of the higher moments of the density matrix [53].

The global protocol serves as a proof of principle that higher-point OTOCs contain new scrambling dynamics not present in $C_{4}(t)$. This motivates the development of protocols based on classical shadows [35] in the following section, which only utilize single-qubit, random Clifford unitaries.

\section{CLASSICAL SHADOW PROTOCOLS}

We present three protocols to estimate $C_{4 k}(t)$ using classical shadows generated through quantum shadow tomography $[34,35]$. We summarize the essentials of shadow tomography, but refer to [35] for further details.

\section{A. Shadow tomography}

The classical shadow protocols rely on the prediction of functions of an $N$-qubit state $\rho$. First, define a random unitary as a tensor product of local unitaries,

$$
U=\bigotimes_{i=1}^{N} u_{i}
$$

Each single-qubit unitary $u_{i}$ is drawn randomly and independently from the Clifford group. Evolve $\rho$ to $U \rho U^{\dagger}$. Upon measurement in the computational basis, the state collapses to $|\hat{b}\rangle\langle\hat{b}|$ by Born's rule, where $|\hat{b}\rangle \in\{0,1\}^{\otimes N}$ is an $N$-bit random variable. That is, the state is randomly measured in the Pauli basis, similar to traditional tomography. In shadow tomography, however, one is interested in estimating the properties of the state, not in reconstructing it. After measurement, the outcome is stored and processed classically. Then, we classically compute $U^{\dagger}|\hat{b}\rangle\langle\hat{b}| U$ and apply the inverted channel $\mathcal{M}^{-1}=\bigotimes_{i=1}^{N} \mathcal{M}_{i}^{-1}$, where $\mathcal{M}_{i}^{-1}(\cdot)=3(\cdot)-\operatorname{Tr}(\cdot) I$ and $I$ is the identity on a single qubit. The result is a classical snapshot of $\rho$,

$$
\hat{\rho}(U ; \hat{b})=\mathcal{M}^{-1}\left(U^{\dagger}|\hat{b}\rangle\langle\hat{b}| U\right) .
$$

The classical snapshot satisfies $\mathbb{E}(\hat{\rho}(U ; \hat{b}))=\rho$, where $\mathbb{E}$ is the average of the outcomes and of the unitaries over the local Clifford group. More formally,

$$
\begin{aligned}
\mathbb{E}(\hat{\rho}(U ; \hat{b}))= & \mathbb{E}_{U \sim \mathrm{Cl}(2)^{\otimes N}}\left(\sum_{b \in\{0,1\}^{N}}\left\langle b\left|U \rho U^{\dagger}\right| b\right\rangle\right. \\
& \left.\times \mathcal{M}^{-1}\left(U^{\dagger}|b\rangle\langle b| U\right)\right) .
\end{aligned}
$$

As a result, an observable $O$ can be estimated using the snapshot through $\operatorname{Tr}\{O \hat{\rho}\}$ with the average value $\mathbb{E}(\operatorname{Tr}\{O \hat{\rho}\})=$ $\operatorname{Tr}\{O \mathbb{E}(\hat{\rho})\}=\operatorname{Tr}\{O \rho\}$. To reduce the statistical error of the estimation, one can repeat this process to generate $K$ independent classical snapshots. The classical shadow of $\rho$ is defined as the set of these snapshots

$$
S(\rho, K)=\left\{\hat{\rho}^{(i)}\left(U_{i} ; \hat{b}_{i}\right)\right\}_{i=1}^{K} .
$$

Each snapshot corresponds to a new measurement. $K$ is referred to as the size of the shadow. The shadow can also be used to estimate nonlinear functions of $\rho$, which are encountered in our measurement protocols. As an example, a second-order function $f_{2}$ can be written as $f_{2}(\rho)=\operatorname{Tr}\left\{O^{\prime} \rho^{\otimes 2}\right\}$ for some observable $O^{\prime}$ on $\mathcal{H}_{d}^{\otimes 2}$. This can be estimated by using two distinct snapshots: $\operatorname{Tr}\left\{O^{\prime} \hat{\rho} \otimes \hat{\rho}^{\prime}\right\}$.

\section{B. Multi-Bell-state protocol}

We construct a protocol to measure $C_{4 k}(t)$ by preparing multiple Bell states. Since $C_{4 k}(t)$ is a function of the evolution unitary $U_{H}(t)$, we introduce Bell states to "store" $U_{H}(t)$ $[67,68]$. Consider a $2 N$-qubit system. Let $\rho_{\text {Bell }}=|\Phi\rangle\langle\Phi|$ be the maximally entangled state, where

$$
|\Phi\rangle=\frac{1}{d^{1 / 2}} \sum_{i=1}^{d}|i\rangle \otimes|i\rangle
$$

and $|i\rangle \in \mathcal{H}_{d}$. State $|\Phi\rangle$ consists of $N$ Bell states. To simplify computations, we introduce a graphical calculus [48] and refer to Appendix B for its complete description. With some stylistic adaptation from Ref. [69], $\rho_{\text {Bell }}$ is expressed as

$$
\rho_{\text {Bell }}=\frac{1}{d} . \square .
$$

Evolving $\rho_{\text {Bell }}$ with the unitary channel associated with $U_{H} \otimes$ $I_{N}$, where $I_{N}$ is the identity on $N$ qubits, the resulting state and its diagram are, respectively,

$$
\rho_{H}=\left(U_{H} \otimes I_{N}\right) \rho_{\mathrm{Bell}}\left(U_{H}^{\dagger} \otimes I_{N}\right),
$$




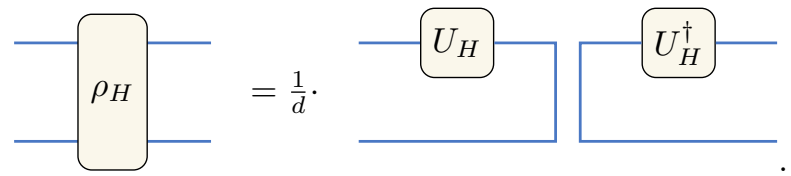

The time dependence of $\rho_{H}(t)$ is suppressed for conciseness. State $\rho_{H}$ is actually the channel-state duality for $U_{H}(t)$.

Now write $C_{4 k}(t)$ in terms of $\rho_{H}$. First, express the correlator diagrammatically,

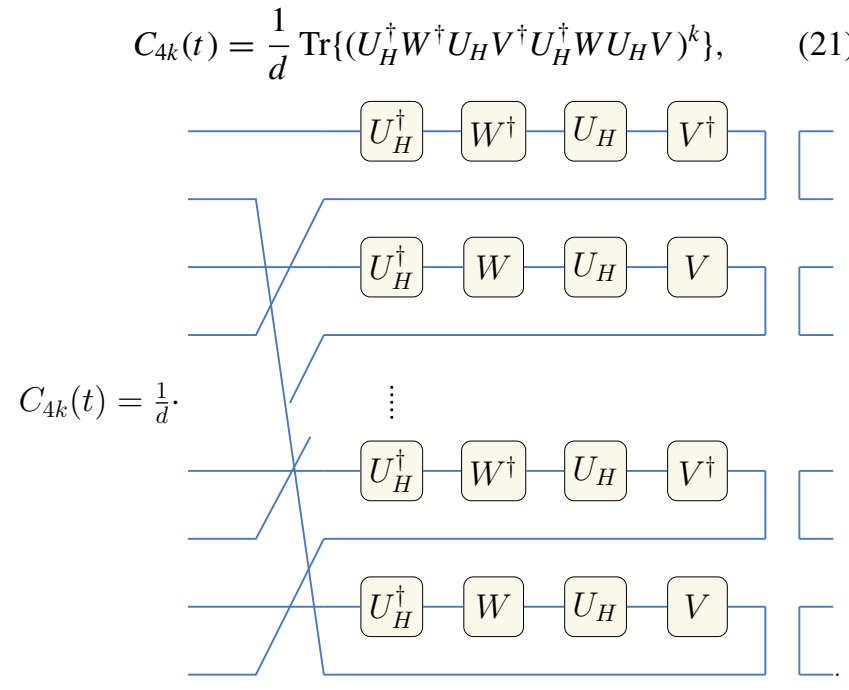

"Slide" $U_{H}^{\dagger}$ leftwards and use the implied periodic boundary conditions. Introduce the following identity relating $A_{1}$ to its transpose:

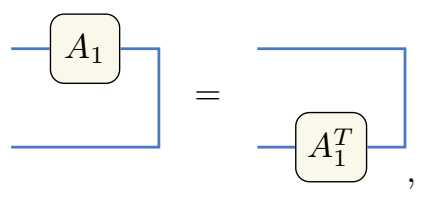

for $A_{1}=V, V^{\dagger}$. The correlator is now drawn as

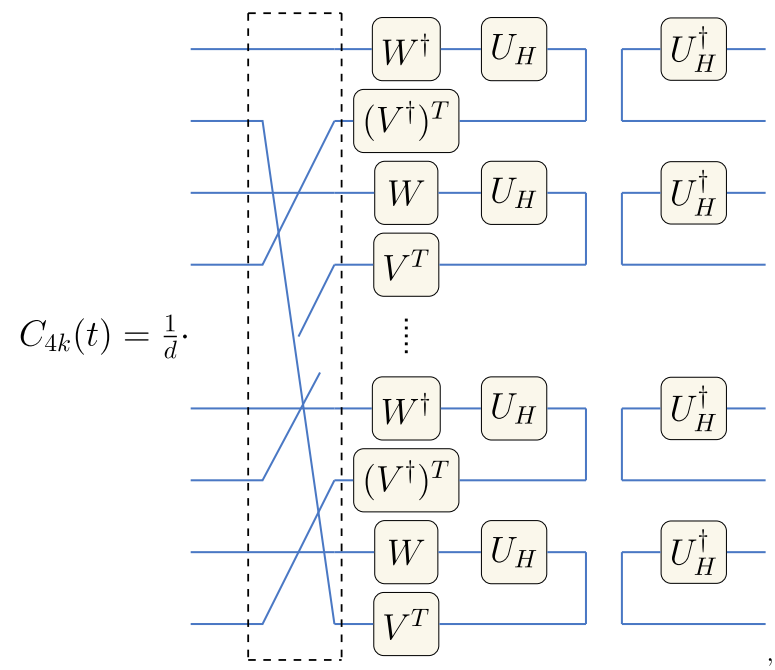

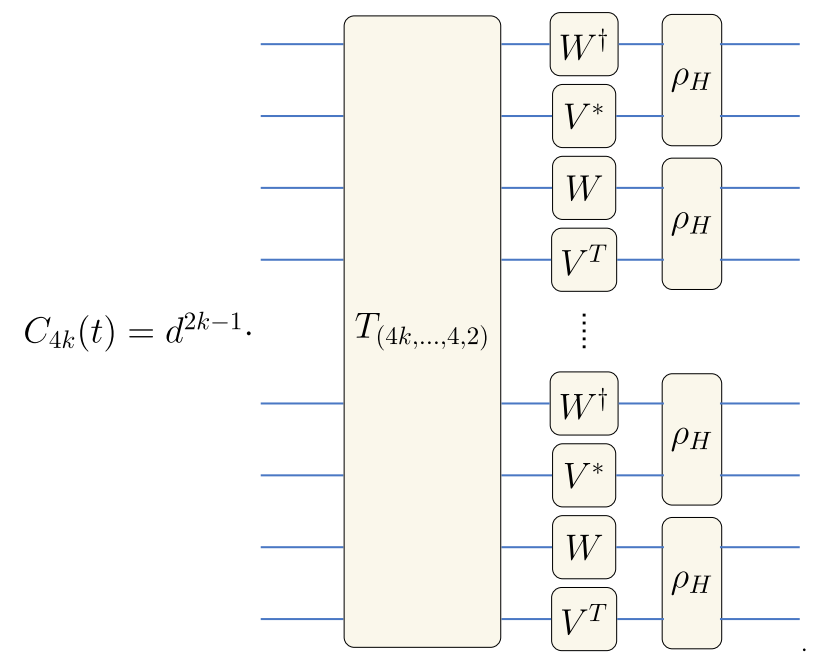

(25)

The dashed rectangle in Eq. (24) is the cyclic permutation operator over even indexes, $T_{(4 k, \ldots, 4,2)}$. The correlator is now

$$
C_{4 k}(t)=d^{2 k-1} \operatorname{Tr}\left\{O_{4 k} \rho_{H}^{\otimes 2 k}\right\},
$$

where $O_{4 k}=T_{(4 k, \ldots, 4,2)}\left(W^{\dagger} \otimes V^{*} \otimes W \otimes V^{T}\right)^{\otimes k}$. $O_{4 k}$ has no time dependence; all time dependence is stored in $\rho_{H}$. $C_{4 k}(t)$ can therefore be estimated by performing shadow tomography on $\rho_{H}$.

One can also define a general $4 k$-point correlator as an expectation value over an arbitrary state $\rho$ :

$$
\left\langle\left(W^{\dagger}(t) V^{\dagger} W(t) V\right)^{k}\right\rangle_{\rho} \equiv \operatorname{Tr}\left\{\rho\left(W^{\dagger}(t) V^{\dagger} W(t) V\right)^{k}\right\} .
$$

This can also be expressed in terms of $\rho_{H}$ :

$$
\begin{aligned}
\left\langle\left(W^{\dagger}(t) V^{\dagger} W(t) V\right)^{k}\right\rangle_{\rho}= & d^{2 k} \operatorname{Tr}\left\{T_{(4 k, \ldots, 4,2)}\left(I^{\otimes(4 k-1)} \otimes \rho^{T}\right)\right. \\
& \times\left(W^{\dagger} \otimes V^{*} \otimes W \otimes V^{T}\right)^{\otimes k} \\
& \left.\times \rho_{H}^{\otimes 2 k}\right\} .
\end{aligned}
$$

The appearance of $\rho^{T}$ may seem alarming, since the transpose is not a physical operation. However, since only $\rho_{H}$ is measured, $\rho^{T}$ is treated as an ordinary operator. This correlator enables an analysis of scrambling beyond the high temperature thermal state. Although our protocol can measure this general correlator, we focus on the maximally mixed state.

We use shadow tomography to construct an estimator for $C_{4 k}(t)$. First, construct a classical shadow of size $K \geqslant 2 k$ for state $\rho_{H}$ :

$$
S_{H}\left(\rho_{H}, K\right)=\left\{\hat{\rho}_{H}^{(i)}\left(U_{i} ; \hat{b}_{i}\right)\right\}_{i=1}^{K} .
$$

We suppress the arguments of each snapshot and write $\hat{\rho}_{H}^{(i)}=$ $\hat{\rho}_{H}^{(i)}\left(U_{i} ; \hat{b}_{i}\right)$. Using the classical shadow, construct an unbiased estimator for $C_{4 k}(t)$ through the $U$-statistic [42,70]:

$\hat{C}_{4 k}(t)=\frac{d^{2 k-1}}{(2 k) !}\left(\begin{array}{c}K \\ 2 k\end{array}\right)^{-1} \sum_{i_{1} \neq i_{2} \neq \cdots \neq i_{2 k}} \operatorname{Tr}\left\{O_{4 k} \hat{\rho}_{H}^{\left(i_{1}\right)} \otimes \cdots \otimes \hat{\rho}_{H}^{\left(i_{2 k}\right)}\right\}$.

The sum is carried out over all size $2 k$ permutations of classical snapshots in $S_{H}$. Each term in the sum is a function of $2 k$ 


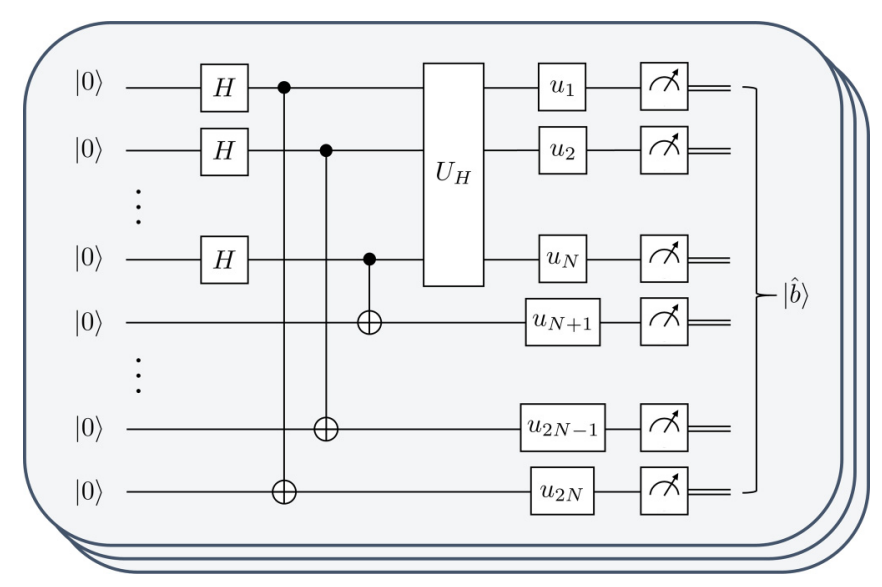

FIG. 1. Quantum circuit to generate a classical snapshot for the multi-Bell-state protocol. $N$ Bell states are prepared. The subsystem composed of one qubit from each Bell state is evolved by $U_{H}(t)$. Each time this circuit runs, $u_{i}$ is randomly sampled anew from the Clifford group. A classical shadow is generated by running this circuit $K$ times.

independent snapshots. Unitary $U_{i_{j}}$ and outcome $\hat{b}_{i_{j}}$ of snapshot $\hat{\rho}_{H}^{\left(i_{j}\right)}$ are independent of the unitaries and outcomes of any other snapshot $\hat{\rho}_{H}^{\left(i_{j^{\prime}}\right)}$. When averaging $\hat{C}_{4 k}(t)$ over all Clifford unitaries and outcomes, each $\hat{\rho}_{H}^{\left(i_{j}\right)}$ is averaged individually:

$$
\mathbb{E}\left(\hat{C}_{4 k}(t)\right)=\frac{d^{2 k-1}}{(2 k) !}\left(\begin{array}{c}
K \\
2 k
\end{array}\right)^{-1} \sum_{i_{1} \neq \cdots \neq i_{2 k}} \operatorname{Tr}\left\{O_{4 k} \bigotimes_{j=1}^{2 k} \mathbb{E}\left(\hat{\rho}_{H}^{\left(i_{j}\right)}\right)\right\} .
$$

Since all snapshots satisfy $\mathbb{E}\left(\hat{\rho}_{H}^{\left(i_{j}\right)}\right)=\rho_{H}$, then $\mathbb{E}\left(\hat{C}_{4 k}(t)\right)=$ $C_{4 k}(t)$. Thus, $\hat{C}_{4 k}(t)$ is an unbiased estimator of $C_{4 k}(t)$.

We summarize the multi-Bell-state protocol (see Fig. 1) to estimate $C_{4 k}(t)$ :

(1) Prepare $N$ Bell states.

(2) Evolve the subsystem consisting of one qubit from each Bell state with $U_{H}(t)$.

(3) Create a classical shadow of size of $K \geqslant 2 k$ for this state.

(4) Use the shadow post-processing in Eq. (30) to compute $\hat{C}_{4 k}(t)$.

The initial state can be readily prepared. For instance, experiments with Rydberg-atom qubits and ultracold atoms have demonstrated high-fidelity control of many pairs of Bell states [71,72]. The multi-Bell-state protocol carries the advantage that no additional assumptions aside from unitarity and locality are made about $W$ or $V$. Furthermore, single-qubit Clifford unitaries can readily be implemented in experiments. The protocol can also be extended to measure a general correlator for an arbitrary state. Although the OTOC corresponds to an expectation value over an $N$-qubit state, this protocol requires a measurement of $2 N$ qubits. For systems limited in size, a protocol requiring a measurement of only $N$ qubits without a preparation of Bell states is favorable. We develop such a protocol in the next section.

\section{Mixed state protocol}

We introduce a protocol to estimate $C_{4 k}(t)$ requiring a measurement on only $N$ qubits, by writing the operator $V$ in terms of some initial state. Using the cyclic property of the trace, the correlator can be written as

$$
C_{4 k}(t)=\frac{1}{d} \operatorname{Tr}\left\{\left(U_{H}(t) V U_{H}^{\dagger}(t) W U_{H}(t) V U_{H}^{\dagger}(t) W\right)^{k}\right\} .
$$

Set $W$ and $V$, for example, to

$$
W=Z \otimes I^{\otimes N-1}, \quad V=I^{\otimes N-1} \otimes Z .
$$

Write $Z=2|0\rangle\langle 0|-I$ and introduce the initial state

$$
\rho_{\text {in }}=\frac{1}{2^{N-1}} I^{\otimes N-1} \otimes|0\rangle\langle 0|,
$$

such that $V=d \rho_{\text {in }}-I^{\otimes N}$. Defining the time-evolved state

$$
\rho_{V}=U_{H}(t) \rho_{i n} U_{H}^{\dagger}(t)
$$

and, using the expression for $V$, the correlator becomes

$$
C_{4 k}(t)=\frac{1}{d} \operatorname{Tr}\left\{\left(d^{2} \rho_{V} W \rho_{V} W-d \rho_{V}-d W \rho_{V} W+I^{\otimes N}\right)^{k}\right\} .
$$

Shadow tomography can be used to construct an estimator for $C_{4 k}(t)$ for any $k$. For demonstration, we estimate the fourpoint and eight-point OTOCs. Evaluating Eq. (36) at $k=1$,

$$
\begin{aligned}
C_{4}(t) & =d \operatorname{Tr}\left\{\rho_{V} W \rho_{V} W\right\}-1 \\
& =d \operatorname{Tr}\left\{\rho_{V}^{\otimes 2} W^{\otimes 2} T_{(1,2)}\right\}-1 .
\end{aligned}
$$

By creating a shadow of size $K \geqslant 2$ for state $\rho_{V}$,

$$
S_{V}\left(\rho_{V}, K\right)=\left\{\hat{\rho}_{V}^{(i)}\left(U_{i} ; \hat{b}_{i}\right)\right\}_{i=1}^{K},
$$

an unbiased estimator of $C_{4}(t)$ can be constructed through the $U$-statistic:

$$
\hat{C}_{4}(t)=\frac{1}{2}\left(\begin{array}{c}
K \\
2
\end{array}\right)^{-1} \sum_{i_{1} \neq i_{2}} d \operatorname{Tr}\left\{\hat{\rho}_{V}^{\left(i_{1}\right)} \otimes \hat{\rho}_{V}^{\left(i_{2}\right)} W^{\otimes 2} T_{(1,2)}\right\}-1 .
$$

The sum is taken over all size 2 permutations of snapshots in $S_{V}$.

Equation (36) evaluated at $k=2$ yields the eight-point OTOC,

$$
\begin{aligned}
C_{8}(t)= & d^{3} \operatorname{Tr}\left\{\rho_{V} W \rho_{V} W \rho_{V} W \rho_{V} W\right\} \\
& -4 d^{2} \operatorname{Tr}\left\{\rho_{V} W \rho_{V} W \rho_{V}\right\} \\
& +4 d \operatorname{Tr}\left\{\rho_{V} W \rho_{V} W\right\}+1 .
\end{aligned}
$$

Noting $\rho_{V}^{2}=\frac{2}{d} \rho_{V}$, the correlator simplifies to

$$
C_{8}(t)=L_{8}(t)-4 C_{4}(t)-3,
$$

with the leading-order term

$$
L_{8}(t)=d^{3} \operatorname{Tr}\left\{\rho_{V} W \rho_{V} W \rho_{V} W \rho_{V} W\right\}
$$

which determines the scrambling dynamics not captured by the four-point correlator. Using shadow $S_{V}$ of size $K \geqslant 4$, the 


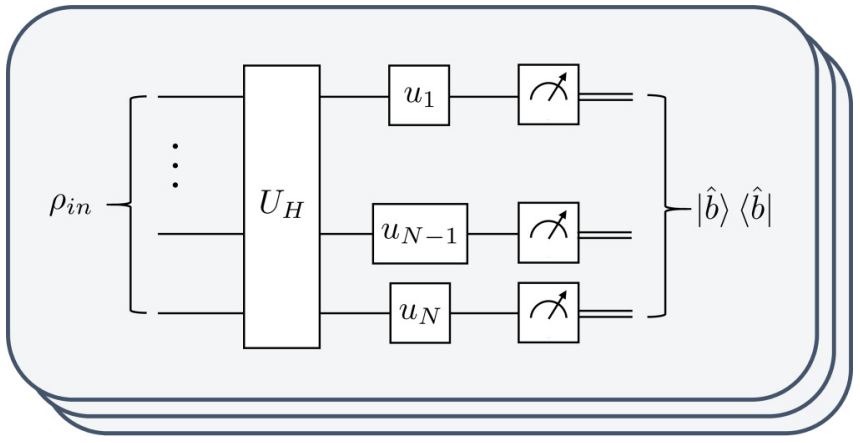

FIG. 2. Quantum circuit which generates a classical snapshot in the mixed state protocol. The initial state $\rho_{i n}=\frac{1}{2^{N-1}} I^{\otimes N-1} \otimes|0\rangle\langle 0|$ evolves with $U_{H}(t)$, then local random Clifford unitaries $u_{i}$ act on each qubit, followed by computational basis measurement.

unbiased estimator for the leading-order term is

$$
\hat{L}_{8}(t)=\frac{1}{4 !}\left(\begin{array}{c}
K \\
4
\end{array}\right)^{-1} \sum_{\substack{i_{1} \neq i_{2} \\
\neq i_{3} \neq i_{4}}} d^{3} \operatorname{Tr}\left\{\left(\bigotimes_{j=1}^{4} \hat{\rho}_{V}^{\left(i_{j}\right)}\right) W^{\otimes 4} T_{(1,2,3,4)}\right\} .
$$

The sum is over all size 4 permutations of snapshots in $S_{V}$. Thus, the unbiased estimator for the eight-point correlator is

$$
\hat{C}_{8}(t)=\hat{L}_{8}(t)-4 \hat{C}_{4}(t)-3,
$$

where $\hat{C}_{4}(t)$ is given by Eq. (39).

The mixed state protocol (see Fig. 2) to estimate $C_{4 k}(t)$ is as follows:

(1) Prepare state $\frac{1}{2^{N-1}} I^{\otimes N-1} \otimes|0\rangle\langle 0|$, where qubit $N$ is in $|0\rangle\langle 0|$ and the remaining qubits are in the maximally mixed state.

(2) Evolve with $U_{H}(t)$.

(3) Create a shadow of size $K \geqslant 2 k$ for the state.

(4) Use the shadow to compute $\hat{C}_{4 k}(t)$.

The advantage of the mixed state protocol over the multiBell-state protocol is a measurement of only $N$ qubits is required and a preparation of Bell states is avoided. Initial state $\rho_{\text {in }}$ can be prepared on a nuclear magnetic resonance quantum simulator [25].

\section{Single-Bell-state protocol}

As a hybrid of the two protocols developed previously, we construct a protocol which introduces one Bell state by using just one ancillary qubit. The advantage of this protocol is that the operator $V$ is not predetermined by the initial input state as in Sec. III C, but is selected in the final classical post-processing stage.

For simplicity, we first compute the estimator for $C_{4}(t)$, then generalize the result to $C_{4 k}(t)$. For instance, by taking $W=Z_{1}$ and $V=X_{N}$, which both act on $\mathcal{H}_{d}$, the four-point correlator in Eq. (32) is

$$
C_{4}(t)=\frac{1}{d} \operatorname{Tr}\left\{U_{H}(t) X_{N} U_{H}^{\dagger}(t) Z_{1} U_{H}(t) X_{N} U_{H}^{\dagger}(t) Z_{1}\right\} .
$$

Introduce a diagram for the correlator where each leg now represents an index for the single-qubit Hilbert space $\mathcal{H}_{2}$. A slash mark on a leg denotes all remaining qubits. The diagram with periodic boundary conditions is

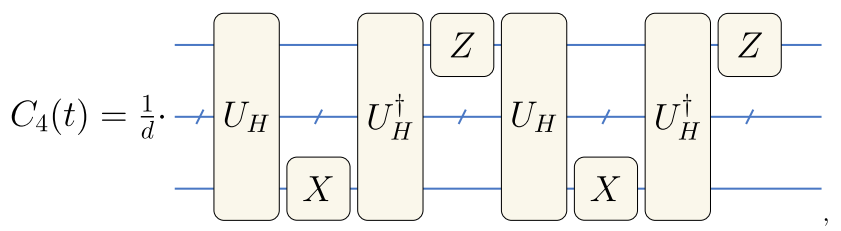

(46)

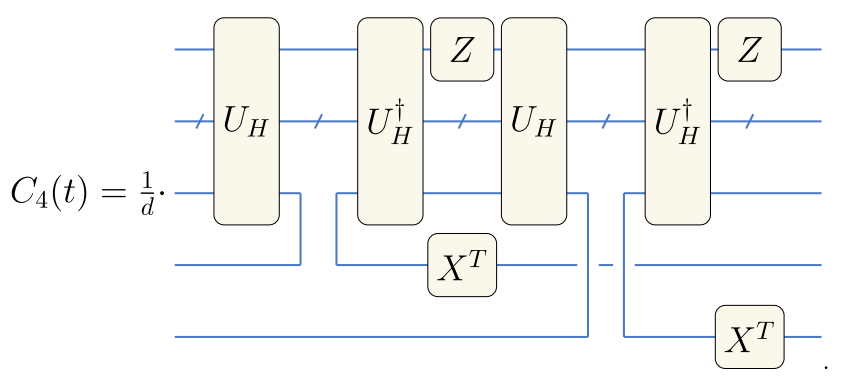

To simplify notation, define $B \equiv Z_{1} X_{a_{1}}^{T}$ with Pauli $Z$ on system qubit 1 and $X^{T}$ on an ancillary qubit denoted by $a_{1}$. The correlator is redrawn as

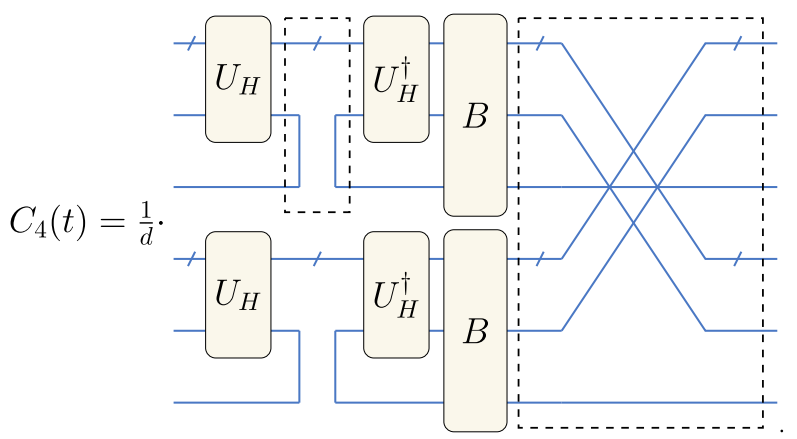

(48)

To clarify, the slash marks in Eq. (47) and (48) represent $N-2$ and $N-1$ system qubits, respectively. Define $\rho_{N, a_{1}}$ as the initial state where the $N$ th system qubit forms a Bell state with the ancillary qubit. The remaining $N-1$ system qubits are in the maximally mixed state. The small dotted rectangle in Eq. (48) represents $\rho_{N, a_{1}}$ up to a normalization factor $d$. The large dotted rectangle represents the permutation operator $\prod_{l=1}^{N} T_{(l, N+1+l)}$, which is a product of swap operators. Define the time-evolved state

$$
\rho_{H, N, a_{1}}=\left(U_{H} \otimes I_{a_{1}}\right) \rho_{N, a_{1}}\left(U_{H}^{\dagger} \otimes I_{a_{1}}\right),
$$

where $I_{a_{1}}$ is the identity on the ancillary qubit. The correlator is

$$
C_{4}(t)=d \cdot \operatorname{Tr}\left\{\left(\rho_{H, N, a_{1}} \otimes \rho_{H, N, a_{1}}\right) B^{\otimes 2} \prod_{l=1}^{N} T_{(l, N+1+l)}\right\} .
$$

To construct an estimator for $C_{4}(t)$, create a shadow of size $K \geqslant 2$ for state $\rho_{H, N, a_{1}}$ :

$$
S_{S B}\left(\rho_{H, N, a_{1}}, K\right)=\left\{\hat{\rho}_{H, N, a_{1}}^{(i)}\left(U_{i} ; \hat{b}_{i}\right)\right\}_{i=1}^{K} .
$$




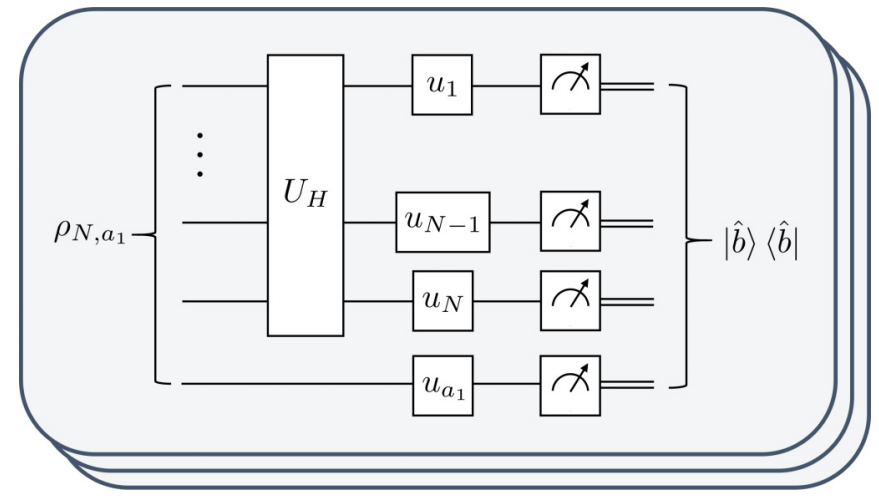

FIG. 3. Quantum circuit which generates a classical snapshot for the single-Bell-state protocol. The initial state is $\rho_{N, a_{1}}$, where system qubit $N$ forms a Bell state with an ancillary qubit, while the remaining qubits are in the maximally mixed state. Unitary $U_{H}(t)$ evolves the $N$ system qubits. Then local random Clifford unitaries $u_{i}$ act on each qubit, followed by a measurement.

Use this shadow to compute

$$
\begin{aligned}
\hat{C}_{4}(t)= & \frac{d}{2}\left(\begin{array}{c}
K \\
2
\end{array}\right)^{-1} \sum_{i \neq j} \operatorname{Tr}\left\{\left(\hat{\rho}_{H, N, a_{1}}^{(i)} \otimes \hat{\rho}_{H, N, a_{1}}^{(j)}\right)\right. \\
& \left.\times B^{\otimes 2} \prod_{l=1}^{N} T_{(l, N+1+l)}\right\} .
\end{aligned}
$$

This procedure can be generalized to construct an estimator for $C_{4 k}(t)$ for $k \geqslant 2$ :

$$
\begin{aligned}
\hat{C}_{4 k}(t)= & \frac{d^{2 k-1}}{(2 k) !}\left(\begin{array}{c}
K \\
2 k
\end{array}\right)^{-1} \sum_{i_{1} \neq \cdots \neq i_{2 k}} \operatorname{Tr}\left\{\left(\otimes_{j=1}^{2 k} \hat{\rho}_{H, N, a_{1}}^{\left(i_{j}\right)}\right) B^{\otimes 2 k}\right. \\
& \left.\times \prod_{l=1}^{N} T_{(l, N+1+l, \ldots,(2 k-1)(N+1)+l)}\right\} .
\end{aligned}
$$

The sum is over all size $2 k$ permutations of snapshots in $S_{S B}$.

The single-Bell-state protocol (see Fig. 3) to estimate $C_{4 k}(t)$ is

(1) Prepare $N$ system qubits and 1 ancillary qubit. Create a Bell state between system qubit $N$ and the ancillary qubit. Prepare the remaining system qubits in the maximally mixed state.

(2) Evolve the system qubits with $U_{H}(t)$.

(3) Construct a shadow of size $K \geqslant 2 k$ for the state.

(4) Use the shadow to compute $\hat{C}_{4 k}$.

The single-Bell-state protocol carries the advantage that the OTOCs are given by a single trace, rather than a sum of traces as in the mixed state protocol. This feature makes the single-Bell-state protocol more appropriate for the commutator type correlators discussed in Appendix E3. This protocol is ideal for systems with a limited number of qubits, since it only introduces one ancillary qubit. Similar to the multi-Bell-state protocol, a manipulation of diagrams can easily yield an estimator for a general $4 k$-point correlator over an arbitrary state. The same classical shadow can be used to compute OTOCs for different choices of $W$ and $V$, allowing for the calculation of multiple OTOCs with the same batch of measurements.

\section{STATISTICAL ERROR ANALYSIS}

We analyze the statistical error for estimators due to the finite shadow size $K$. We focus on the mixed state protocol from Sec. III C for its experimental practicality. We state the following results on the variance in shadow tomography:

Fact 1. (Proposition 3 in [35]). For a state $\rho \in \mathcal{H}_{d}$ and a linear function $\operatorname{Tr}(O \rho)$, the single-shot variance of the function obeys

$$
\operatorname{Var}[\operatorname{Tr}\{O \hat{\rho}\}] \leqslant d \operatorname{Tr}\left(O^{2}\right),
$$

where $\hat{\rho}$ is a snapshot of $\rho$.

Lemma 1. For a state $\rho^{\otimes 2} \in \mathcal{H}_{d}^{\otimes 2}$ and a nonlinear function $\operatorname{Tr}\left\{O_{2} \rho^{\otimes 2}\right\}$ with observable $O_{2}=T_{(1,2)} W^{\otimes 2}$, the single-shot variance of the function obeys

$$
\operatorname{Var}\left[\operatorname{Tr}\left\{O_{2} \hat{\rho} \otimes \hat{\rho}^{\prime}\right\}\right] \leqslant d^{3},
$$

where $W$ is any Pauli operator and $\hat{\rho}, \hat{\rho}^{\prime}$ are two distinct snapshots of $\rho$.

The proof of Lemma 1 is in Appendix F1. The lemma shows a significant enhancement when compared with using Fact 1 on the doubled Hilbert space, which yields $d^{2} \operatorname{Tr}\left\{O_{2}^{2}\right\}=$ $d^{2} \operatorname{Tr}\left\{T_{(1,2)}^{2}\right\}=d^{4}$. Lemma 1 is also suitable for $O_{2}=T_{(1,2)}$, which can tighten the variance for the purity measurement performed in $[35,42]$. The improvement relies on the prior knowledge that the target state here is in the tensor product form $\rho \otimes \rho$. We believe a modification of Lemma 1 with an appropriate $O$ on a few-copy state can enhance shadow tomography in other scenarios.

\section{A. Variance of four-point OTOC}

We compute the variance of $\hat{C}_{4}(t)$ in Eq. (39). The summation index constraint $i_{1} \neq i_{2}$ in $\hat{C}_{4}(t)$ can be changed to $i_{1}<i_{2}$ by symmetrizing the observable as follows

$$
\begin{aligned}
\hat{C}_{4}(t)= & \frac{d}{2}\left(\begin{array}{l}
K \\
2
\end{array}\right)^{-1} \sum_{i_{1} \neq i_{2}} \operatorname{Tr}\left\{\hat{\rho}_{V}^{\left(i_{1}\right)} \otimes \hat{\rho}_{V}^{\left(i_{2}\right)} W^{\otimes 2} T_{(1,2)}\right\}-1 \\
= & d\left(\begin{array}{l}
K \\
2
\end{array}\right)^{-1} \sum_{i_{1}<i_{2}} \operatorname{Tr}\left\{\frac{W^{\otimes 2} T_{(1,2)}+T_{(1,2)} W^{\otimes 2}}{2}\right. \\
& \left.\times \hat{\rho}_{V}^{\left(i_{1}\right)} \otimes \hat{\rho}_{V}^{\left(i_{2}\right)}\right\}-1 \\
= & d\left(\begin{array}{l}
K \\
2
\end{array}\right)^{-1} \sum_{i_{1}<i_{2}} \operatorname{Tr}\left\{T_{(1,2)} W^{\otimes 2} \hat{\rho}_{V}^{\left(i_{1}\right)} \otimes \hat{\rho}_{V}^{\left(i_{2}\right)}\right\}-1 \\
= & d\left(\begin{array}{c}
K \\
2
\end{array}\right)^{-1} \sum_{i_{1}<i_{2}} \hat{D}_{4}\left(i_{1}, i_{2}\right)-1 .
\end{aligned}
$$

Define $\hat{D}_{4}\left(i_{1}, i_{2}\right)=\operatorname{Tr}\left\{T_{(1,2)} W^{\otimes 2} \hat{\rho}_{V}^{\left(i_{1}\right)} \otimes \hat{\rho}_{V}^{\left(i_{2}\right)}\right\}$. The third line is due to $T_{(1,2)}$ commuting with $W^{\otimes 2}$. To simplify notation, we suppress all time dependence in this section. The variance of $\hat{C}_{4}$ satisfies

$$
\operatorname{Var}\left(\hat{C}_{4}\right)=\left[d\left(\begin{array}{l}
K \\
2
\end{array}\right)^{-1}\right]^{2} \operatorname{Var}\left(\sum_{i_{1}<i_{2}} \hat{D}_{4}\left(i_{1}, i_{2}\right)\right),
$$


with

$$
\begin{aligned}
& \operatorname{Var}\left(\sum_{i_{1}<i_{2}} \hat{D}_{4}\left(i_{1}, i_{2}\right)\right) \\
& =\mathbb{E}\left[\left(\sum_{i_{1}<i_{2}} \hat{D}_{4}\left(i_{1}, i_{2}\right)\right)^{2}\right]-\mathbb{E}\left[\sum_{i_{1}<i_{2}} \hat{D}_{4}\left(i_{1}, i_{2}\right)\right]^{2} \\
& =\sum_{\substack{i_{1}<i_{2}, j_{1}<j_{2}}}\left\{\mathbb{E}\left[\hat{D}_{4}\left(i_{1}, i_{2}\right) \hat{D}_{4}\left(j_{1}, j_{2}\right)\right]-D_{4}^{2}\right\} \\
& =\sum_{\substack{i_{1}<i_{2}, j_{1}<j_{2}}}\left[V_{4}\left(i_{1}, i_{2}, j_{1}, j_{2}\right)-D_{4}^{2}\right] .
\end{aligned}
$$

Define $D_{4}=\mathbb{E}\left[\hat{D}_{4}\left(i_{1}, i_{2}\right)\right]$ for any $i_{1}, i_{2}$ and $V_{4}\left(i_{1}, i_{2}, j_{1}, j_{2}\right)=$ $\mathbb{E}\left[\hat{D}_{4}\left(i_{1}, i_{2}\right) \hat{D}_{4}\left(j_{1}, j_{2}\right)\right] . V_{4}$ depends on the coincidences between indices $i_{1}, i_{2}$ and $j_{1}, j_{2}$, respectively. A coincidence indicates that the two $\hat{D}_{4}$ in the second line of Eq. (58) share the same snapshot, and thus are not independent. There are three possible coincidence cases of the indices discussed as follows:

(i) No coincidence: in this case $V_{4}$ simplifies to $V_{4}=$ $\mathbb{E}\left[\hat{D}_{4}\left(i_{1}, i_{2}\right)\right] \mathbb{E}\left[\hat{D}_{4}\left(j_{1}, j_{2}\right)\right]=D_{4}^{2}$ since all snapshots are independent.

(ii) One coincidence: take, for example, the case $i_{1} \neq j_{1}$ and $i_{2}=j_{2}$. There are a total of $2\left(\begin{array}{c}K \\ 1\end{array}\right)\left(\begin{array}{c}R-1 \\ 2\end{array}\right)$ such terms. We simplify

$$
\begin{aligned}
V_{4}= & \mathbb{E}\left[\operatorname{Tr}\left\{T_{(1,2)} W^{\otimes 2} \hat{\rho}_{V}^{\left(i_{1}\right)} \otimes \hat{\rho}_{V}^{\left(i_{2}\right)}\right\}\right. \\
& \left.\times \operatorname{Tr}\left\{T_{(1,2)} W^{\otimes 2} \hat{\rho}_{V}^{\left(j_{1}\right)} \otimes \hat{\rho}_{V}^{\left(i_{2}\right)}\right\}\right] \\
= & \mathbb{E}\left[\operatorname{Tr}\left\{T_{(1,2)} W^{\otimes 2} \rho_{V} \otimes \hat{\rho}_{V}\right\}^{2}\right] \\
= & \mathbb{E}\left[\operatorname{Tr}\left\{W \rho_{V} W \hat{\rho}_{V}\right\}^{2}\right] .
\end{aligned}
$$

In the second line, we take the expectation of $\hat{\rho}_{V}^{\left(i_{1}\right)}$ and $\hat{\rho}_{V}^{\left(j_{1}\right)}$, then denote $\hat{\rho}_{V}^{\left(i_{2}\right)}$ as $\hat{\rho}_{V}$ without ambiguity. Set $O_{1}=$ $W \rho_{V} W$ so that $\mathbb{E}\left[\operatorname{Tr}\left\{O_{1} \hat{\rho}_{V}\right\}\right]=D_{4}$. We can analyze $V_{4}-$ $D_{4}^{2}=\operatorname{Var}\left[\operatorname{Tr}\left\{O_{1} \hat{\rho}_{V}\right\}\right]$ using Fact 1 .

(iii) Two coincidences: $i_{1}=j_{1}$ and $i_{2}=j_{2}$. There are $\left(\begin{array}{c}K \\ 2\end{array}\right)$ such terms in total. We can write

$$
V_{4}=\mathbb{E}\left[\operatorname{Tr}\left\{T_{(1,2)} W^{\otimes 2} \hat{\rho}_{V} \otimes \hat{\rho}_{V}^{\prime}\right\}^{2}\right],
$$

where $\hat{\rho}_{V}$ and $\hat{\rho}_{V}^{\prime}$ are independent snapshots. Set $O_{2}=$ $T_{(1,2)} W^{\otimes 2}$ for state $\rho_{V}^{\otimes 2} \in \mathcal{H}_{d}^{\otimes 2}$ so that Lemme 1 bounds $V_{4}-$ $D_{4}^{2}=\operatorname{Var}\left[\operatorname{Tr}\left\{O_{2} \hat{\rho}_{V} \otimes \hat{\rho}_{V}^{\prime}\right\}\right]$.

Inserting the above three cases into Eq. (58),

$$
\begin{aligned}
\operatorname{Var}\left(\hat{C}_{4}\right)= & d^{2}\left(\begin{array}{l}
K \\
2
\end{array}\right)^{-2} \operatorname{Var}\left(\sum_{i_{1}<i_{2}} \hat{D}_{4}\left(i_{1}, i_{2}\right)\right) \\
= & d^{2}\left(\begin{array}{c}
K \\
2
\end{array}\right)^{-1}\left\{2(K-2) \operatorname{Var}\left[\operatorname{Tr}\left\{O_{1} \hat{\rho}_{V}\right\}\right]\right. \\
& \left.+\operatorname{Var}\left[\operatorname{Tr}\left\{O_{2} \hat{\rho}_{V} \otimes \hat{\rho}_{V}^{\prime}\right\}\right]\right\} \\
\leqslant & d^{2}\left[\frac{4(K-2)}{K(K-1)} \operatorname{Tr}\left\{O_{1}^{2}\right\} d+\frac{2 d^{3}}{K(K-1)}\right]
\end{aligned}
$$

$$
\begin{aligned}
& =d^{2}\left[\frac{8(K-2)}{K(K-1)}+\frac{2 d^{3}}{K(K-1)}\right] \\
& \leqslant \frac{8 d^{2}}{K}+\frac{3 d^{5}}{K^{2}} .
\end{aligned}
$$

In the third line, we apply Fact 1 and Lemma 1 to the variances respectively. The fourth line is due to $\operatorname{Tr}\left\{O_{1}^{2}\right\}=\operatorname{Tr}\left\{\rho_{V}^{2}\right\}=\frac{2}{d}$. By using Chebyshev's inequality, one arrives at

Proposition 1. To estimate the four-point correlator $C_{4}=$ $d \operatorname{Tr}\left\{T_{(1,2)} W^{\otimes 2} \rho_{V}^{\otimes 2}\right\}-1$ under confidence level $\delta$ and error $\epsilon$, the shadow size $K$ satisfying

$$
K \geqslant 2 \max \left\{\frac{8 d^{2}}{\epsilon^{2} \delta}, \frac{\sqrt{3} d^{2.5}}{\epsilon \sqrt{\delta}}\right\}
$$

is sufficient to let $\operatorname{Prob}\left(\left|C_{4}-\hat{C}_{4}\right| \leqslant \epsilon\right) \geqslant 1-\delta$.

Remark 1. One may apply full quantum state tomography $[73,74]$ on $\rho_{V}$ to directly calculate $C_{4}=$ $d \operatorname{Tr}\left\{T_{(1,2)} W^{\otimes 2} \rho_{V}^{\otimes 2}\right\}-1$. To make the error of $C_{4}$ less than $\epsilon$, the error on the state $\rho_{V}$ should be around $\epsilon^{\prime}=\epsilon / d$. As a result, the necessary number of measurements on $\rho_{V}$ with quantum tomography is $K=\Omega\left(d^{2} / \epsilon^{\prime 2}\right)=\Omega\left(d^{4} / \epsilon^{2}\right)$. Even with the optimistic estimation of taking the trace distance to be comparable to the infidelity, $K=\Omega\left(d^{2} / \epsilon^{\prime}\right)=\Omega\left(d^{3} / \epsilon\right)$, which is still worse than Eq. (62). Furthermore, if one is restricted to independent measurements on a single copy of $\rho_{V}$ (like in the classical shadow protocols), the scaling worsens: $K=\Omega\left(d^{3} / \epsilon^{2}\right)=\Omega\left(d^{5} / \epsilon^{2}\right)$.

Note that one can enhance the scaling analysis of $K$ with respect to the confidence level $\delta$ to $\log (1 / \delta)$ in Eq. (62) by using the median-of-means technique [35].

\section{B. Variance of eight-point OTOC}

We compute the variance of the unbiased estimator in Eq. (44). By equivalently symmetrizing the observable, the estimator is

$$
\begin{aligned}
\hat{C}_{8}= & d^{3}\left(\begin{array}{c}
K \\
4
\end{array}\right)^{-1} \sum_{\substack{i_{1}<i_{2} \\
<i_{3}<i_{4}}} \operatorname{Tr}\left\{\Theta_{4}\left(T_{(1,2,3,4)} W^{\otimes 4}\right) \bigotimes_{j=1}^{4} \hat{\rho}_{V}^{\left(i_{j}\right)}\right\} \\
& -4 d\left(\begin{array}{c}
K \\
2
\end{array}\right)^{-1} \sum_{\substack{j_{1}<j_{2}\\
}} \operatorname{Tr}\left\{\Theta_{2}\left(T_{(1,2)} W^{\otimes 2}\right) \hat{\rho}_{V}^{\left(j_{1}\right)} \otimes \hat{\rho}_{V}^{\left(j_{2}\right)}\right\}+1 \\
= & d^{3}\left(\begin{array}{c}
K \\
4
\end{array}\right)^{-1} \sum_{\substack{i_{1}<i_{2} \\
<i_{3}<i_{4}}} \hat{D}_{8}\left(i_{1}, i_{2}, i_{3}, i_{4}\right) \\
& -4 d\left(\begin{array}{c}
K \\
2
\end{array}\right)^{-1} \sum_{j_{1}<j_{2}} \hat{D}_{4}\left(j_{1}, j_{2}\right)+1
\end{aligned}
$$

where $\hat{D}_{8}\left(i_{1}, i_{2}, i_{3}, i_{4}\right)$ and $\hat{D}_{4}\left(j_{1}, j_{2}\right)$ denote the random variables under the summations,

$$
\Theta_{t}(\cdot)=\frac{1}{t !} \sum_{\pi \in S_{t}} T_{\pi}(\cdot) T_{\pi^{-1}}
$$

is the twirling channel of the symmetry group on $\mathcal{H}_{d}^{\otimes t}$, and $T_{\pi}$ is the permutation operator for permutation $\pi$. $\Theta_{t}\left(T_{(1,2, \ldots, t)} W^{\otimes t}\right)=\Theta_{t}\left(T_{(1,2, \ldots, t)}\right) W^{\otimes t}$, as $W^{\otimes t}$ commutes with all $T_{\pi}$. Since permutation $(1,2, \ldots, t)$ is an element 
of $S_{t}$, the twirling $\Phi_{t}\left(T_{(1,2, \ldots, t)}\right)$ returns the average on the elements in the same conjugate class of $T_{(1,2, \ldots, t)}$. For the $t=2$ case, $\Phi_{t}\left(T_{(1,2)}\right)=T_{(1,2)}$. For $t=4$, there are six elements in this class, so one need only consider six permutations of the snapshots: $\left\{i_{1} i_{2} i_{3} i_{4}, i_{1} i_{2} i_{4} i_{3}, i_{1} i_{3} i_{2} i_{4}, i_{1} i_{3} i_{4} i_{2}, i_{1} i_{4} i_{2} i_{3}, i_{1} i_{4} i_{3} i_{2}\right\}$. This improves the classical computation time when calculating $\hat{C}_{8}(t)$.

Similar to the analysis of $\hat{C}_{4}$ in Sec. IV A, the variance $\operatorname{Var}\left(\hat{C}_{8}\right)=\mathbb{E}\left[\hat{C}_{8}^{2}\right]-\mathbb{E}\left[\hat{C}_{8}\right]^{2}$ depends on the coincidences of the indices. For simplicity, we consider the leading contribution to $\operatorname{Var}\left(\hat{C}_{8}\right)$, namely, the variance of the first term $\hat{L}_{8}$ :

$$
\begin{aligned}
\operatorname{Var}\left(\hat{L}_{8}\right) & =\left[d^{3}\left(\begin{array}{l}
K \\
4
\end{array}\right)^{-1}\right]^{2} \operatorname{Var}\left(\sum_{\vec{i}} \hat{D}_{8}(\vec{i})\right) \\
& =d^{6}\left(\begin{array}{l}
K \\
4
\end{array}\right)^{-2} \sum_{\vec{i}, \vec{j}}\left\{\mathbb{E}\left[\hat{D}_{8}(\vec{i}) \hat{D}_{8}(\vec{j})\right]-D_{8}^{2}\right\} \\
& =d^{6}\left(\begin{array}{c}
K \\
4
\end{array}\right)^{-2} \sum_{\vec{i}, \vec{j}}\left[V_{8}(\vec{i}, \vec{j})-D_{8}^{2}\right] .
\end{aligned}
$$

The summation over $\vec{i}$ labels the summation over indices $i_{1}<i_{2}<i_{3}<i_{4}$, and similarly for $\vec{j}$. Define $V_{8}(\vec{i}, \vec{j})=$ $\mathbb{E}\left[\hat{D}_{8}(\vec{i}) \hat{D}_{8}(\vec{j})\right]$. There are subleading terms like $\mathbb{E}\left[\hat{D}_{8} \hat{D}_{4}\right]-$ $\mathbb{E}\left[\hat{D}_{8}\right] \mathbb{E}\left[\hat{D}_{4}\right]$ and $\operatorname{Var}\left(\hat{D}_{4}\right)$ contributing to $\operatorname{Var}\left(\hat{C}_{8}\right)$, and they can be calculated similarly. As in the four-point case, we consider the coincidences of $\vec{i}, \vec{j}$. We present the result, but leave the derivation in Appendix F2.

Proposition 2. The variance of the estimator of the leading term $\hat{L}_{8}(t)$ for the eight-point OTOC in Eq. (44) can be upper bounded by

$$
\begin{aligned}
\operatorname{Var}\left(\hat{L}_{8}\right) \leqslant & \frac{64 d^{5} D_{8}}{K}+\frac{16\left(4 d D_{4}+d^{2} D_{4}^{2}+8 D_{2}^{2}+2\right)}{K^{2}} \\
& +\frac{32\left[d^{10}\left(1+D_{2}^{2}\right)+3 d^{8}\right]}{K^{3}}+\frac{4\left(d^{14}+5 d^{6}\right)}{K^{4}},
\end{aligned}
$$

where $D_{4 k}=\operatorname{Tr}\left\{\left(W \rho_{V}\right)^{2 k}\right\}$. In the early-time limit, the bound becomes

$$
\frac{512 d^{2}}{K}+\frac{352}{K^{2}}+\frac{32\left(2 d^{10}+3 d^{8}\right)}{K^{3}}+\frac{4\left(d^{14}+5 d^{6}\right)}{K^{4}} .
$$

The variance behaves like $O\left(d^{14} / K^{4}\right)$ and still outperforms full quantum state tomography (see Appendix F2). We apply Fact 1 in the proof of Proposition 2, but expect an improvement for large coincidence cases by using similar techniques as Lemma 1.

\section{NUMERICAL SIMULATIONS}

We present simulations of predicted and estimated OTOCs using an $N$-qubit mixed-field Ising spin chain with Hamiltonian

$$
H=-\frac{1}{E_{0}}\left(J \sum_{i=1}^{N-1} Z_{i} Z_{i+1}+h_{x} \sum_{i=1}^{N} X_{i}+h_{z} \sum_{i=1}^{N} Z_{i}\right) .
$$

The parameters are $J=1, h_{x}=1.05, h_{z}=0.5$, and $E_{0}=$ $\sqrt{4 J^{2}+2 h_{x}^{2}+2 h_{z}^{2}} . X_{i}$ and $Z_{i}$ are Pauli operators on the $i$ th

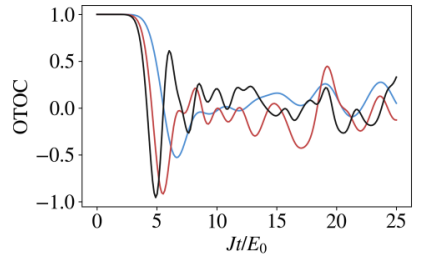

(a)

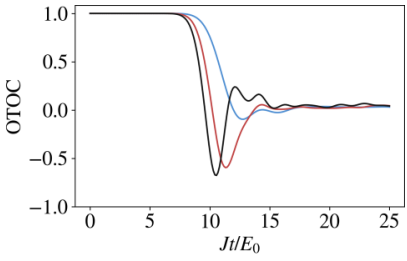

(b)

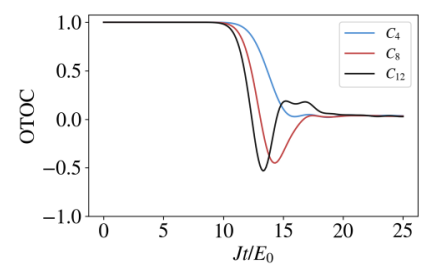

(c)

FIG. 4. Predicted values of the first three OTOCs for qubit numbers (a) $N=4$, (b) $N=8$, and (c) $N=10$ for a mixed-field Ising spin chain. In all cases, $W=Z_{1}$ and $V=Z_{N}$.

qubit. This nonintegrable model has been shown to exhibit chaotic behavior [11].

We simulate the evolution of the correlator $C_{4 k}(t)=$ $\left\langle\left(W^{\dagger}(t) V^{\dagger} W(t) V\right)^{k}\right\rangle$, where $W=Z_{1}$ and $V=Z_{N}$, for the first three OTOCs $(k=1,2,3)$ and various $N$ in Fig. 4 . In the early-time limit, all OTOCs experience an initial steep decay. As the qubit number increases, the time taken for any OTOC to exhibit this decay increases. Since the separation between $W$ and $V$ is larger, $W$ takes longer to spread to the support of $V$, delaying the growth of [ $W(t), V]$. Intuitively, since all OTOCs are related to a Schatten $2 n$-norm of this commutator, the decay of every OTOC is delayed. Referring to the $N=8$ and $N=10$ cases of Fig. 4, all OTOCs decay to the same value in the large-time limit, reflecting the system's degree of scrambling. This point is discussed further in Appendix E2. These three characteristics: an initial steep decay, the delay in decay for larger qubit systems, and the large-time decay to a floor value (for sufficiently large systems) are well-known features of the famous four-point OTOC for chaotic systems. Higher-point OTOCs also display these characteristics, making them reliable standalone quantities to study scrambling.

Studying the early-time behavior of OTOCs sheds light on the initial rate of information delocalization [75-77]. In Fig. 5, we numerically simulate the eight-point correlator's leadingorder term $L_{8}(t)$ from Eq. (42) for various Ising spin-chain
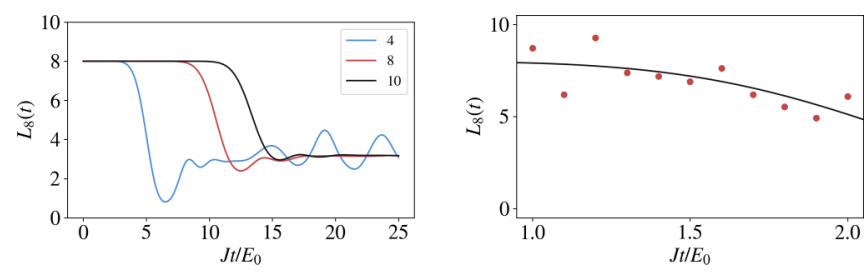

FIG. 5. Left: Dynamics of the predicted leading-order term $L_{8}(t)$ for $N=4,8,10$. Right: Early-time behavior of the predicted leading-order term $L_{8}(t)$ (black line) plotted against its estimator $\hat{L}_{8}(t)$ (red dots) using the mixed state protocol for a two-qubit chain. Each point represents an average over 25 estimators, each computed with a different shadow of size 150 . 


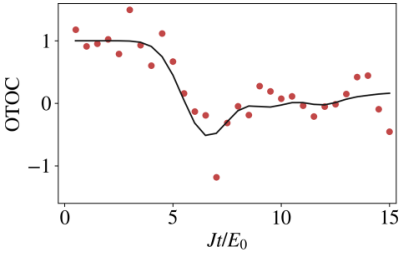

(a)

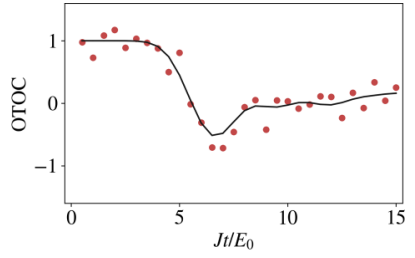

(b)

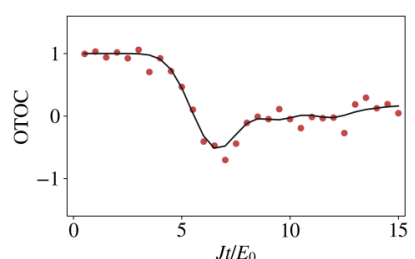

(c)

FIG. 6. Predicted four-point OTOC $C_{4}(t)$ (black line) plotted against its estimator $\hat{C}_{4}(t)$ (red dots) constructed from the mixed state protocol for shadow sizes of (a) $K=5000$, (b) $K=10000$, and (c) $K=15000$. A mixed-field Ising spin chain of $N=4$ qubits is used.

lengths. The early-time dynamics of this term contribute to the faster initial decay of the eight-point correlator relative to the four-point OTOC in Fig. 4. This is consistent with the discussion in Appendix $\mathrm{A}$ in which the multiple perturbations found in higher-point OTOCs are expected to result in faster information delocalization. In Fig. 5, we also simulate the measurement of $\hat{L}_{8}(t)$ from Eq. (43) with the mixed state protocol. To reduce the post-processing computations, we run the protocol with a fixed shadow size $K$ several times and average the results. In Fig. 6, the predicted four-point OTOC is plotted against its estimator from Eq. (39) using the mixed state protocol for various shadow sizes. As the shadow size increases, agreement between the two improves.

\section{CONCLUSION}

We present a definition of higher-point out-of-time-ordered correlators to describe the dynamics of quantum scrambling in chaotic systems. In the early-time limit, higher-point correlators exhibit faster decay and reveal information delocalization earlier than the four-point OTOC. We present protocols using classical shadows to estimate these correlators and show they can outperform full quantum state tomography. For sufficiently many measurements, good agreement between the predicted and estimated values can be achieved. The protocols avoid time reversal and can probe the dynamics of OTOCs at any time. They can be implemented using singlequbit Pauli measurements, making them ideal for experiments with single-qubit control. The protocols here extract nonlinear functions by measuring only a single copy of a target state, thus avoiding the preparation of multiple identical copies and the joint control and readout on them. In addition, the same classical shadow can be used to compute multiple correlators by using classical post-processing. Furthermore, the protocols can be used to construct estimators for general correlators over an arbitrary initial state $\left\langle(W(t) V W(t) V)^{k}\right\rangle_{\rho}$, allowing for the study of OTOCs beyond the thermal state background.
There are a few interesting points which merit further investigation. First, our protocols can be directly extended to the noisy evolution scenario, where the system dynamics are described by general quantum channels. Adopting noise mitigation methods [78,79] may be an intriguing approach to distinguish quantum scrambling from classical decoherence effects [80-82]. Second, the enhancement of the variance analysis based on prior knowledge of the state's tensor structure can be generalized to other scenarios, which can further improve the practicality of shadow tomography for nonlinear functions. Third, it is possible to extend the protocols to measure other quantities in quantum scrambling, such as the operator weight distribution [83-85]. Finally, it is important to explore the operational and physical implications of higherpoint OTOCs, and demonstrate our protocols on near-term quantum platforms.

\section{ACKNOWLEDGMENTS}

We would like to thank Beni Yoshida for helpful discussions on noncommutator type correlators, Xun Gao for discussions on diagrammatic representations of quantum objects, and Pei Zeng for discussions on shadow tomography. We also thank the anonymous referees for their helpful comments. This work was supported in part by ARO Grants No. W911NF-19-1-0302 and No. W911NF20-1-0082. Y.Z. was also supported by National Research Foundation of Singapore under its NRF-ANR joint program (NRF2017-NRF-ANR004 VanQuTe), the Quantum Engineering Program QEP-SP3, the Singapore Ministry of Education Tier 1 grant RG162/19, FQXi-RFP-IPW-1903 from the foundational Questions Institute and Fetzer Franklin Fund, a donor advised fund of Silicon Valley Community Foundation, and the Zhongguancun Haihua Institute for Frontier Information Technology. Any opinions, findings and conclusions or recommendations expressed in this material are those of the author(s) and do not reflect the views of the National Research Foundation, Singapore.

The Appendix is organized as follows. In Appendix A, we give an interpretation of higher-point OTOCs in terms of a perturbation evolution procedure. The basics of tensor network representation and random matrix theory are given in Appendix B and Appendix C, respectively. In Appendix D, we relate the correlators to global random unitaries and thus prove the validity of the protocol shown in Sec. II. In Appendix E, we study the late-time behavior of higher-point OTOCs by using the Haar random unitary ensemble, and show that our protocol can also be adopted to measure commutator-type correlators defined in Ref. [33]. The proof of Lemma 1 is given in Appendix F1, which enhances the variance analysis based on the product structure of the target state. The proof of Proposition 2 for the variance of the eight-point OTOC is given in Appendix F2.

\section{APPENDIX A: INTERPRETATION OF HIGHER-POINT OTOCs}

We propose a physical interpretation of higher-point OTOCs. For simplicity, we approximate the expectation value over the maximally mixed state as one over a Haar random 


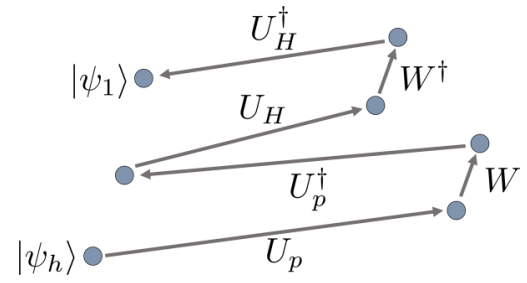

FIG. 7. Schematic of a single perturbed evolution procedure evolving $\left|\psi_{h}\right\rangle$ to $\left|\psi_{1}\right\rangle$.

state $\left|\psi_{h}\right\rangle[86,87]$. The approximate four-point OTOC is

$$
\begin{aligned}
\tilde{C}_{4}(t) & =\left\langle\psi_{h}\left|W^{\dagger}(t) V^{\dagger} W(t) V\right| \psi_{h}\right\rangle \\
& =\left\langle\psi_{h}\left|U_{H}^{\dagger} W^{\dagger} U_{H} V^{\dagger} U_{H}^{\dagger} W U_{H} V\right| \psi_{h}\right\rangle .
\end{aligned}
$$

In analogy to the Loschmidt echo $[88,89]$, define the perturbed evolution unitary as $U_{p}(t)=U_{H}(t) V$. The state $U_{p}(t)\left|\psi_{h}\right\rangle$ represents a small "kick," $V$, perturbing $\left|\psi_{h}\right\rangle$ before evolution by $U_{H}(t)$. The approximate OTOC in terms of $U_{p}(t)$ is

$$
\tilde{C}_{4}(t)=\left\langle\psi_{h}\left|U_{H}^{\dagger} W^{\dagger} U_{H} U_{p}^{\dagger} W U_{p}\right| \psi_{h}\right\rangle .
$$

The state $\left|\psi_{1}\right\rangle=U_{H}^{\dagger} W^{\dagger} U_{H} U_{p}^{\dagger} W U_{p}\left|\psi_{h}\right\rangle$ is interpreted through following perturbation procedure (see Fig. 7). Evolve $\left|\psi_{h}\right\rangle$ by $U_{p}(t)$, then apply $W$. Evolve the state using $U_{p}^{\dagger}(t)$, which corresponds to backwards time evolution by $U_{H}^{\dagger}(t)=U_{H}(-t)$ followed by $V^{\dagger}$. Evolve with $U_{H}(t)$, apply $W^{\dagger}$, and finally evolve backwards in time using $U_{H}^{\dagger}(t)$. The correlator is the overlap between the initial state $\left|\psi_{h}\right\rangle$ and the evolved state $\left|\psi_{1}\right\rangle$. The role of $W$ is to perturb the state and ensure $U_{p}(t)$ is not undone by $U_{p}^{\dagger}(t) . V$ probes how chaotic $U_{H}(t)$ is. In the case where $V$ is the identity, $U_{p}(t)=U_{H}(t)$ and the evolution procedure evolves $\left|\psi_{h}\right\rangle$ away from and back to itself. No chaos is detected. For a nontrivial $V$, the perturbation procedure evolves the state away from $\left|\psi_{h}\right\rangle . \tilde{C}_{4}(t)$ indicates the sensitivity of the evolution of $U_{H}(t)$ to perturbations.

Consider the approximate $4 k$-point OTOC,

$$
\tilde{C}_{4 k}(t)=\left\langle\psi_{h}\left|\left(U_{H}^{\dagger} W^{\dagger} U_{H} U_{p}^{\dagger} W U_{p}\right)^{k}\right| \psi_{h}\right\rangle .
$$

This correlator gives the overlap of $\left|\psi_{h}\right\rangle$ with the state resulting from $k$ applications of the perturbation procedure to $\left|\psi_{h}\right\rangle$. Each application evolves the state successively further from $\left|\psi_{h}\right\rangle$. The higher-point correlators correspond to a larger number of perturbations to the initial state, which probe the chaos induced by $U_{H}(t)$ in finer detail.

We return to the discussion of exact OTOCs. A higherpoint OTOC $C_{4 k}(t)$ reveals the finer scrambling dynamics due to $k$ perturbations by $V$. After each perturbation, evolution by $U_{H}(t)$ further delocalizes the quantum information of the initially local operator $W$. Higher-point correlators therefore scramble information more quickly, resulting in a faster OTOC decay. In a different context, an interpretation of higher-point correlators is given in terms of multiple shock waves [32].

\section{APPENDIX B: GRAPHICAL CALCULUS}

We present a graphical calculus for matrices adapted from $[48,69]$ and mention a related picture-language for quantum information $[68,90]$. An operator $A_{1}$ is drawn as a box with input and output legs:

$$
A_{1}=-A_{1} .
$$

The purpose of the coloring is solely to distinguish these diagrams from quantum circuits. By expanding $A_{1}$ in a basis, $A_{1}=\sum_{i, j} a_{i, j}^{1}|i\rangle\langle j|$, we let the left leg correspond to $|i\rangle$ and the right leg correspond to $\langle j|$. Unless otherwise stated, each leg index corresponds to a state in Hilbert space $\mathcal{H}_{d}$. The transpose of $A_{1}$ is represented by

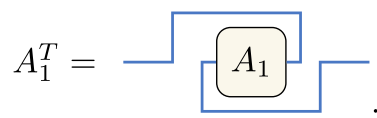

A product of two operators is drawn by connecting the output leg of one with the input leg of the other:

$$
A_{1} A_{2}=A_{1} A_{2} \text {. }
$$

More formally, a connection between legs is an index contraction and hence represents matrix multiplication.

The trace $\operatorname{Tr}\left\{A_{1}\right\}=\sum_{i} a_{i, i}^{1}\langle i \mid i\rangle$ is drawn by connecting the legs of $A_{1}$ :

$$
\operatorname{Tr}\left\{A_{1}\right\}=A_{1} .
$$

A tensor product of operators is represented as a "stacking" of boxes:

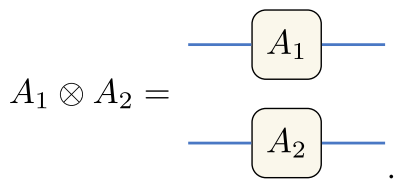

The trace of this tensor product is drawn by connecting the input and output legs at each level. For a permutation $\pi$, we may define the permutation operator $T_{\pi}$ through

$$
\begin{aligned}
& T_{\pi}\left|a_{1}\right\rangle \otimes\left|a_{2}\right\rangle \otimes \cdots \otimes\left|a_{k}\right\rangle \\
& \quad=\left|a_{\pi(1)}\right\rangle \otimes\left|a_{\pi(2)}\right\rangle \otimes \cdots \otimes\left|a_{\pi(k)}\right\rangle .
\end{aligned}
$$

For concreteness, consider the permutation $\pi=(1)(2,3)$ and a tensor product of three states $\left|a_{1}\right\rangle \otimes\left|a_{2}\right\rangle \otimes\left|a_{3}\right\rangle$. The corresponding permutation operator acts as follows:

$$
T_{(1)(2,3)}\left|a_{1}\right\rangle \otimes\left|a_{2}\right\rangle \otimes\left|a_{3}\right\rangle=\left|a_{1}\right\rangle \otimes\left|a_{3}\right\rangle \otimes\left|a_{2}\right\rangle .
$$

Diagrammatically, this permutation operator is

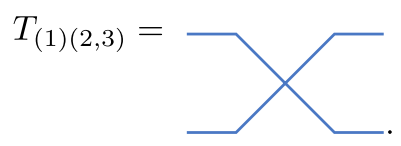


Diagrams for other permutation operators can be constructed in a similar manner. As an example, we compute the following trace:

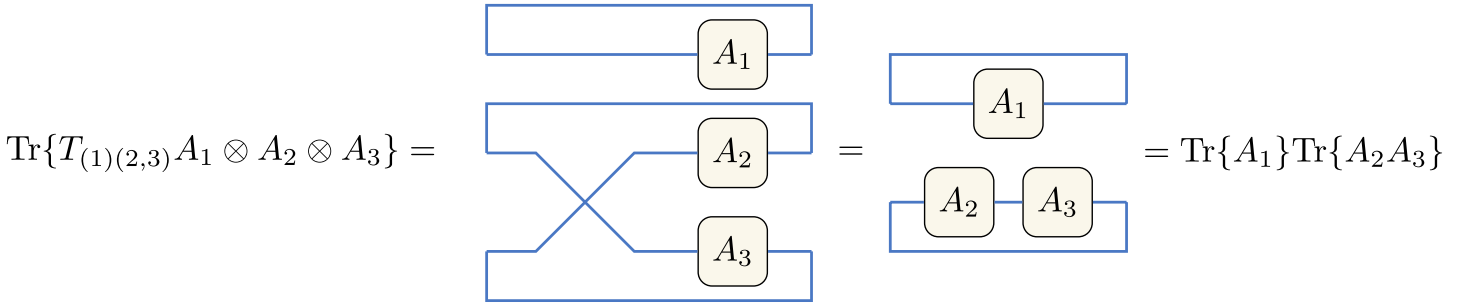

The diagram for the Bell state $|\Phi\rangle=\frac{1}{d^{1 / 2}} \sum_{i=1}^{d}|i\rangle \otimes|i\rangle$ is defined as

$$
|\Phi\rangle=\frac{1}{d^{1 / 2}}
$$

As an outer product, the state is

$$
|\Phi\rangle\langle\Phi|=\frac{1}{d} . \square .
$$

The diagram for $|\Phi\rangle$ can be used to construct the useful identity

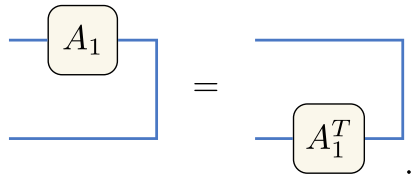

This can be directly verified using the diagrammatic representation of $A_{1}^{T}$ from Eq. (B2).

\section{APPENDIX C: BACKGROUND ON RANDOM MATRIX THEORY}

This section relates permutation operators to random matrices, which allow for the computation of OTOCs through random measurements. For a more complete discussion, see $[33,91]$. Let $U$ be a unitary on Hilbert space $\mathcal{H}_{d}$. Define the operator

$$
A=\bigotimes_{i=1}^{k} A_{i}
$$

where $A_{i}$ is an operator on $\mathcal{H}_{d}$. By the Schur-Weyl duality, if $\left[A, U^{\otimes k}\right]=0$ for all unitaries $U$, then $A$ can be written as a linear combination of permutations operators $T_{\pi}$ :

$$
A=\sum_{\pi \in S_{k}} c_{\pi} T_{\pi}
$$

The sum is carried out over $S_{k}$, the set of all permutations of the set $\{1,2, \ldots, k\}$. Define the $k$-fold twirling channel of $A$ as

$$
\Phi_{\text {Haar }}^{(k)}(A)=\int_{\text {Haar }}\left(U^{\dagger \otimes k}\right) A U^{\otimes k} d U .
$$

The integral is over the Haar measure $d U$ on the unitary group. As the Haar measure is invariant under the action of the group, we write the twirling channel as a superposition of permutation operators:

$$
\Phi_{\text {Haar }}^{(k)}(A)=\sum_{\pi, \sigma \in S_{k}} C_{\pi, \sigma} T_{\pi} \operatorname{Tr}\left\{T_{\sigma} A\right\},
$$

where the coefficients $C_{\pi, \sigma}$ are known as the Weingarten matrix. They satisfy

$$
\left(C^{-1}\right)_{\pi, \sigma}=d^{f(\pi \circ \sigma)} .
$$

where $f(\pi \circ \sigma)$ is the number of cycles of the composition of permutations $\pi$ and $\sigma$.

Fact 2. Let $\left\{A_{i}\right\}_{i=1}^{k}$ be a set of traceless operators $A_{i}$ on Hilbert space $\mathcal{H}_{d}$ of dimension $d$. Let $T_{\sigma}$ be a permutation operator on $\mathcal{H}_{d}^{\otimes k}$ and let $U$ be a Haar random unitary on $\mathcal{H}_{d}$. Then

$$
\begin{aligned}
& \sum_{\sigma \in D_{k}} \operatorname{Tr}\left\{T_{\sigma} A_{1} \otimes \cdots \otimes A_{k}\right\} \\
& \quad=\frac{(d-1+k) !}{(d-1) !} \frac{\left\langle U^{\dagger} A_{1} U\right\rangle_{\rho_{0}} \cdots\left\langle U^{\dagger} A_{k} U\right\rangle_{\rho_{0}}}{(d-}
\end{aligned}
$$

The sum is carried out over $D_{k}$, the set of derangements (permutations with no fixed points) in $S_{k}$. The notation $\langle\cdot\rangle_{\rho_{0}}=$ $\operatorname{Tr}\left\{\rho_{0} \cdot\right\}$ denotes the expectation value over a pure state $\rho_{0} \in$ $\mathcal{H}_{d}$. The notation $\overline{(\cdots)}$ denotes an integral over the Haar measure on the unitary group: $\overline{(\cdots)}=\int_{\text {Haar }}(\cdots) d U$.

Proof. Consider the $k$-fold twirling channel on $\otimes_{i=1}^{k} A_{i}$ :

$$
\begin{aligned}
& \Phi_{\text {Haar }}^{(k)}\left(A_{1} \otimes \cdots \otimes A_{k}\right) \\
& \quad=\int_{\text {Haar }} d U\left(U^{\dagger \otimes k}\right)\left(A_{1} \otimes \cdots \otimes A_{k}\right)\left(U^{\otimes k}\right) .
\end{aligned}
$$

Introducing pure state $\rho_{0}$, we compute the following trace:

$$
\operatorname{Tr}\left\{\rho_{0}^{\otimes k} \Phi_{\text {Haar }}^{(k)}\left(A_{1} \otimes \cdots \otimes A_{k}\right)\right\}=\overline{\left\langle U^{\dagger} A_{1} U\right\rangle_{\rho_{0}} \cdots\left\langle U^{\dagger} A_{k} U\right\rangle_{\rho_{0}}} .
$$

Using Eq. (C4),

$$
\Phi_{\text {Haar }}^{(k)}\left(A_{1} \otimes \cdots \otimes A_{k}\right)=\sum_{\pi, \sigma \in S_{k}} C_{\pi, \sigma} T_{\pi} \operatorname{Tr}\left\{T_{\sigma} A_{1} \otimes \cdots \otimes A_{k}\right\},
$$

so

$$
\begin{aligned}
\operatorname{Tr} & \left\{\rho_{0}^{\otimes k} \Phi_{\text {Haar }}^{(k)}\left(A_{1} \otimes \cdots \otimes A_{k}\right)\right\} \\
& =\sum_{\pi, \sigma \in S_{k}} C_{\pi, \sigma} \operatorname{Tr}\left\{\rho_{0}^{\otimes k} T_{\pi}\right\} \operatorname{Tr}\left\{T_{\sigma} A_{1} \otimes \cdots \otimes A_{k}\right\} .
\end{aligned}
$$


For any $\pi$, we can write

$$
\operatorname{Tr}\left\{\rho_{0}^{\otimes k} T_{\pi}\right\}=\operatorname{Tr}\left\{\rho_{0}^{k}\right\}^{n_{k}} \operatorname{Tr}\left\{\rho_{0}^{k-1}\right\}^{n_{k-1}} \cdots \operatorname{Tr}\left\{\rho_{0}\right\}^{n_{1}},
$$

where $n_{1}, \ldots, n_{k}$ are some integers. Since $\rho_{0}$ is a pure state, $\operatorname{Tr}\left\{\rho_{0}^{m}\right\}=\operatorname{Tr}\left\{\rho_{0}\right\}=1$ for any positive integer $m$. This yields $\operatorname{Tr}\left\{\rho_{0}^{\otimes k} T_{\pi}\right\}=1$. Our expression for the trace then becomes

$$
\begin{aligned}
\operatorname{Tr} & \left\{\rho_{0}^{\otimes k} \Phi_{\text {Haar }}^{(k)}\left(A_{1} \otimes \cdots \otimes A_{k}\right)\right\} \\
& =\sum_{\sigma \in S_{k}} \operatorname{Tr}\left\{T_{\sigma} A_{1} \otimes \cdots \otimes A_{k}\right\} \sum_{\pi \in S_{k}} C_{\pi, \sigma} .
\end{aligned}
$$

It can be shown that the Weingarten matrix satisfies

$$
\sum_{\pi \in S_{k}} C_{\pi, \sigma}=\frac{(d-1) !}{(d-1+k) !}
$$

The trace then becomes

$$
\begin{aligned}
\operatorname{Tr} & \left\{\rho_{0}^{\otimes k} \Phi_{\text {Haar }}^{(k)}\left(A_{1} \otimes \cdots \otimes A_{k}\right)\right\} \\
& =\frac{(d-1) !}{(d-1+k) !} \sum_{\sigma \in S_{k}} \operatorname{Tr}\left\{T_{\sigma} A_{1} \otimes \cdots \otimes A_{k}\right\} .
\end{aligned}
$$

We now have two expressions for $\operatorname{Tr}\left\{\rho_{0}^{\otimes k} \Phi_{\text {Haar }}^{(k)}\left(A_{1} \otimes \cdots \otimes\right.\right.$ $\left.A_{k}\right)$ \}. Together, they yield

$$
\begin{aligned}
\sum_{\sigma \in S_{k}} \operatorname{Tr}\left\{T_{\sigma} A_{1} \otimes \cdots \otimes A_{k}\right\} \\
\quad=\frac{(d-1+k) !}{(d-1) !} \frac{\left\langle U^{\dagger} A_{1} U\right\rangle_{\rho_{0}} \cdots\left\langle U^{\dagger} A_{k} U\right\rangle_{\rho_{0}}}{(d-1}
\end{aligned}
$$

We have not yet used the traceless property of $A_{i}$, so the above equation is valid even for an $A_{i}$ with a nonvanishing trace. We can split the sum over $S_{k}$ into two sums

$$
\begin{aligned}
\sum_{\sigma \in S_{k}} \operatorname{Tr}\left\{T_{\sigma} A_{1} \otimes \cdots \otimes A_{k}\right\}= & \sum_{\sigma \in D_{k}} \operatorname{Tr}\left\{T_{\sigma} A_{1} \otimes \cdots \otimes A_{k}\right\} \\
& +\sum_{\sigma \in F_{k}} \operatorname{Tr}\left\{T_{\sigma} A_{1} \otimes \cdots \otimes A_{k}\right\} .
\end{aligned}
$$

$D_{k}$ is the set of derangements and $F_{k}$ is the set of permutations in $S_{k}$ containing at least one fixed point. For $\sigma \in F_{k}$, the trace $\operatorname{Tr}\left\{T_{\sigma} A_{1} \otimes \cdots \otimes A_{k}\right\}$ introduces at least one trace of the form $\operatorname{Tr}\left\{A_{i}\right\}$, which vanishes since all $A_{i}$ are traceless. The sum over
$F_{k}$ then vanishes and we may write

$$
\sum_{\sigma \in S_{k}} \operatorname{Tr}\left\{T_{\sigma} A_{1} \otimes \cdots \otimes A_{k}\right\}=\sum_{\sigma \in D_{k}} \operatorname{Tr}\left\{T_{\sigma} A_{1} \otimes \cdots \otimes A_{k}\right\} .
$$

This yields

$$
\begin{aligned}
& \sum_{\sigma \in D_{k}} \operatorname{Tr}\left\{T_{\sigma} A_{1} \otimes \cdots \otimes A_{k}\right\} \\
& \quad=\frac{(d-1+k) !}{(d-1) !} \overline{\left\langle U^{\dagger} A_{1} U\right\rangle_{\rho_{0}} \cdots\left\langle U^{\dagger} A_{k} U\right\rangle_{\rho_{0}}},
\end{aligned}
$$

proving the fact.

\section{APPENDIX D: RELATING CORRELATORS TO GLOBAL RANDOM UNITARIES}

The goal of this section is to write the correlators $\left\langle A_{1} A_{2} A_{1} A_{2}\right\rangle$ and $\left\langle A_{1} A_{2}\right\rangle$ (where all $A_{i}$ are traceless, unitary, and Hermitian) in terms of simpler correlators that can be measured experimentally. This is done by introducing random unitaries.

The correlator $\left\langle A_{1} A_{2}\right\rangle$ may be rewritten in terms of a permutation operator:

$$
\left\langle A_{1} A_{2}\right\rangle=\frac{1}{d} \operatorname{Tr}\left\{A_{1} A_{2}\right\}=\frac{1}{d} \operatorname{Tr}\left\{T_{(1,2)} A_{1} \otimes A_{2}\right\} .
$$

This equation can be proved diagrammatically:
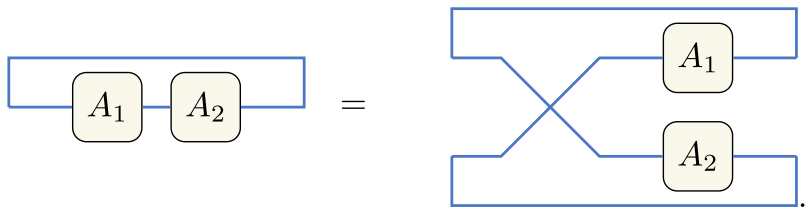

(D2)

Using Fact 2 with $k=2$ yields

$$
\operatorname{Tr}\left\{T_{(1,2)} A_{1} \otimes A_{2}\right\}=d(d+1) \overline{\left\langle U^{\dagger} A_{1} U\right\rangle_{\rho_{0}}\left\langle U^{\dagger} A_{2} U\right\rangle_{\rho_{0}}} .
$$

Note that permutation $(1,2)$ is the only derangement in $D_{2}$. The correlator then becomes

$$
\left\langle A_{1} A_{2}\right\rangle=(d+1) \overline{\left\langle U^{\dagger} A_{1} U\right\rangle_{\rho_{0}}\left\langle U^{\dagger} A_{2} U\right\rangle_{\rho_{0}}} .
$$

The correlators on the right may be measured using the protocol discussed in Sec. II.

We now express $\left\langle A_{1} A_{2} A_{1} A_{2}\right\rangle$ in terms of similar correlators. We begin by introducing a permutation operator

$$
\left\langle A_{1} A_{2} A_{1} A_{2}\right\rangle=\frac{1}{d} \operatorname{Tr}\left\{T_{(1,2,3,4)} A_{1} \otimes A_{2} \otimes A_{1} \otimes A_{2}\right\} .
$$

The application of Fact 2 with $k=4$ simplifies to

$$
\sum_{\sigma \in D_{4}} \operatorname{Tr}\left\{T_{\sigma} A_{1} \otimes A_{2} \otimes A_{1} \otimes A_{2}\right\}=d(d+1)(d+2)(d+3) \overline{\left\langle U^{\dagger} A_{1} U\right\rangle_{\rho_{0}}^{2}\left\langle U^{\dagger} A_{2} U\right\rangle_{\rho_{0}}^{2}} .
$$

The corresponding set of derangements is

$$
\begin{aligned}
D_{4}= & \{(1,2,3,4),(1,2,4,3),(1,3,2,4),(1,3,4,2), \\
& (1,4,2,3),(1,4,3,2),(1,2)(3,4),(1,3)(2,4),(1,4)(2,3)\} .
\end{aligned}
$$


The sum over $D_{4}$ simplifies to

$$
\sum_{\sigma \in D_{4}} \operatorname{Tr}\left\{T_{\sigma} A_{1} \otimes A_{2} \otimes A_{1} \otimes A_{2}\right\}=2 \operatorname{Tr}\left\{A_{1} A_{2} A_{1} A_{2}\right\}+2 \operatorname{Tr}\left\{A_{1} A_{2}\right\}^{2}+\operatorname{Tr}\left\{A_{1}^{2}\right\} \operatorname{Tr}\left\{A_{2}^{2}\right\}+4 \operatorname{Tr}\left\{A_{1}^{2} A_{2}^{2}\right\} .
$$

Since each $A_{i}$ is Hermitian and unitary, $A_{i}^{2}=I$. This yields $\operatorname{Tr}\left\{A_{1}^{2}\right\} \operatorname{Tr}\left\{A_{2}^{2}\right\}=d^{2}$ and $\operatorname{Tr}\left\{A_{1}^{2} A_{2}^{2}\right\}=d$. Note that $\operatorname{Tr}\left\{A_{1} A_{2}\right\}=$ $d\left\langle A_{1} A_{2}\right\rangle$. The sum then simplifies to

$$
\sum_{\sigma \in D_{4}} \operatorname{Tr}\left\{T_{\sigma} A_{1} \otimes A_{2} \otimes A_{1} \otimes A_{2}\right\}=2 \operatorname{Tr}\left\{A_{1} A_{2} A_{1} A_{2}\right\}+2 d^{2}\left\langle A_{1} A_{2}\right\rangle^{2}+d(d+4) .
$$

Equating this to Eq. (D6),

$$
2 \operatorname{Tr}\left\{A_{1} A_{2} A_{1} A_{2}\right\}+2 d^{2}\left\langle A_{1} A_{2}\right\rangle^{2}+d(d+4)=d(d+1)(d+2)(d+3) \overline{\left\langle U^{\dagger} A_{1} U\right\rangle_{\rho_{0}}^{2}\left\langle U^{\dagger} A_{2} U\right\rangle_{\rho_{0}}^{2}} .
$$

Rewriting,

$$
\operatorname{Tr}\left\{A_{1} A_{2} A_{1} A_{2}\right\}=\frac{1}{2} d(d+1)(d+2)(d+3) \overline{\left\langle U^{\dagger} A_{1} U\right\rangle_{\rho_{0}}^{2}\left\langle U^{\dagger} A_{2} U\right\rangle_{\rho_{0}}^{2}}-d^{2}\left\langle A_{1} A_{2}\right\rangle^{2}-\frac{1}{2} d(d+4) .
$$

The correlator then becomes

$$
\left\langle A_{1} A_{2} A_{1} A_{2}\right\rangle=\frac{1}{2}(d+1)(d+2)(d+3) \overline{\left\langle U^{\dagger} A_{1} U\right\rangle_{\rho_{0}}^{2}\left\langle U^{\dagger} A_{2} U\right\rangle_{\rho_{0}}^{2}}-d\left\langle A_{1} A_{2}\right\rangle^{2}-\frac{1}{2}(d+4) .
$$

All correlators on the right can be measured using the protocol in Sec. II.

\section{APPENDIX E: OTOCS AT LATE TIMES}

We calculate the average OTOC values where the evolution unitary is drawn randomly from the Haar measure on the unitary group. Since the Haar random unitary ensemble can be used in place of large-time chaotic evolution, these values serve as a benchmark for the evolution due to physical, chaotic Hamiltonians. Consider the $4 k$-point OTOC

$$
C_{4 k}(t)=\frac{1}{d} \operatorname{Tr}\left\{\left(W^{\dagger}(t) V^{\dagger} W(t) V\right)^{k}\right\},
$$

and take $W$ and $V$ as Pauli operators. For instance, $W=Z_{1} X_{2}$ and $V=Z_{N-1} Z_{N}$. Due to the random feature of the Haar measure, the specific choice of Pauli operators does not affect the result. Based on the Hermitian property of $W$ and $V$, the average OTOC can be written as

$$
\begin{aligned}
\overline{C_{4 k}} & =\int_{\text {Haar }} \frac{1}{d} \operatorname{Tr}\left\{\left[U^{\dagger} W U V\right]^{2 k}\right\} d U \\
& =\frac{1}{d} \operatorname{Tr}\left\{\int_{\text {Haar }}\left[U^{\dagger} W U\right]^{\otimes 2 k} d U V^{\otimes 2 k} T_{\sigma_{0}}\right\} \\
& =\frac{1}{d} \operatorname{Tr}\left\{\Phi_{\text {Haar }}^{(2 k)}\left(W^{\otimes 2 k}\right) V^{\otimes 2 k} T_{\sigma_{0}}\right\} \\
& =\frac{1}{d} \sum_{\pi, \sigma \in S_{2 k}} C_{\pi, \sigma} \operatorname{Tr}\left\{T_{\pi} W^{\otimes 2 k}\right\} \operatorname{Tr}\left\{V^{\otimes 2 k} T_{\sigma^{\prime}}\right\},
\end{aligned}
$$

where in the second line we write the equation over $2 k$ copies of the original Hilbert space $\mathcal{H}_{d}$ and move the integral inside the trace. In the final line we apply the Weingarten formula. We also use the permutations $\sigma_{0}=(1,2, \ldots, 2 k)$ and $\sigma^{\prime}=$ $\sigma_{0} \circ \sigma$. Since any Pauli operator $W$ satisfies $W^{2}=I^{\otimes N}$ and
$\operatorname{Tr}\{W\}=0$, the term $\operatorname{Tr}\left\{T_{\pi} W^{\otimes 2 k}\right\}$ simplifies to

$$
\begin{aligned}
\operatorname{Tr}\left\{T_{\pi} W^{\otimes 2 k}\right\} & =0 & & \text { if } \pi \text { has an odd cycle, } \\
& =d^{f(\pi)} & & \text { if } \pi \text { has no odd cycles, }
\end{aligned}
$$

where $f(\pi)$ is the number of cycles of the permutation $\pi$. For example, (12)(34) and (123)(4) both contain two cycles, but both cycles are odd in the latter case. This relation also holds for $\operatorname{Tr}\left\{V^{\otimes 2 k} T_{\sigma^{\prime}}\right\}$. As a result, Eq. (E2) simplifies to

$$
\overline{C_{4 k}}=\frac{1}{d} \sum_{\substack{\pi, \sigma \in S_{2 k}, \pi, \sigma^{\prime}=\sigma_{0} \circ \sigma \text { even cycles }}} C_{\pi, \sigma} d^{f(\pi)+f\left(\sigma^{\prime}\right)} .
$$

A more general formula is derived in Ref. [33].

\section{Four-point OTOC}

We first compute $\bar{C}_{4}$ where $k=1$. Noting $S_{2}=$ $\{(1)(2),(1,2)\}$, the Weingarten matrix in this case is

$$
C_{\pi, \sigma}=\frac{1}{d^{2}-1}\left(\begin{array}{cc}
1 & -\frac{1}{d} \\
-\frac{1}{d} & 1
\end{array}\right) .
$$

We take $\sigma_{0}=(1,2)$. The sum in Eq. (E4), only allows $\pi=$ $(1,2)$ and $\sigma=(1)(2)$. As a result, the four-point OTOC in the large-time limit satisfies

$$
\overline{C_{4}}=\frac{1}{d} \frac{1}{d^{2}-1}\left(-\frac{1}{d}\right) d^{2}=-\frac{1}{d^{2}-1} .
$$

\section{Eight-point OTOC and (non)commutator types}

Reference [33] has found that there are two general definitions of eight-point OTOCs that display distinct large-time behavior. For operators $A, B, C, D$, one can define the commutator type correlator as $C_{c t}=\langle\tilde{A} B \tilde{C} D \tilde{A} D \tilde{C} B\rangle$ and the noncommutator type correlator as $C_{n c}=\langle\tilde{A} B \tilde{C} D \tilde{A} B \tilde{C} D\rangle$. Define $\tilde{A}=U^{\dagger} A U$, where $U$ is a Haar random unitary. The noncommutator type correlator attains a large-time value which scales as $\sim \frac{1}{d^{2}}$. This occurs even if $U$ is sampled from the Clifford 
ensemble. The four-point OTOC also scales as $\sim \frac{1}{d^{2}}$, indicating that noncommutator type correlators saturate the same floor value as the four-point correlator and therefore cannot reveal any new scrambling information in the large-time limit. The correlator $C_{8}(t)=\langle W(t) V W(t) V W(t) V W(t) V\rangle$ defined in our work is a noncommutator type correlator. Thus, it can only be used to study early-time scrambling dynamics.

The commutator type correlator scales as $\sim \frac{1}{d^{4}}$ at long times, allowing us to probe systems which display higherpoint scrambling in the large-time limit. By taking $A=B$ and $C=D$, the commutator type correlator can be written as

$$
C_{c t}=\langle\tilde{A} A \tilde{C} C \tilde{A} C \tilde{C} A\rangle .
$$

We can adapt our measurement protocols to estimate this correlator.

\section{Estimating commutator type correlators}

Using the technique from Sec. III D, we introduce a single Bell state to compute an estimator for the following commutator type correlator:

$$
\begin{aligned}
C_{c t}(t) & =\langle W(t) W V(t) V W(t) V V(t) W\rangle, \\
& =\frac{1}{d} \operatorname{Tr}\left\{U_{H} W U_{H}^{\dagger} V U_{H} V U_{H}^{\dagger} W U_{H} V U_{H}^{\dagger} V U_{H} W U_{H}^{\dagger} W\right\} .
\end{aligned}
$$

The cyclic property of the trace is used in the second line. Equation (E8) looks similar to the original eight-point OTOC defined in Eq. (7), but displays a different ordering of $W$ and $V$. Taking $W=Z_{1}$ and $V=X_{N}$, the correlator becomes

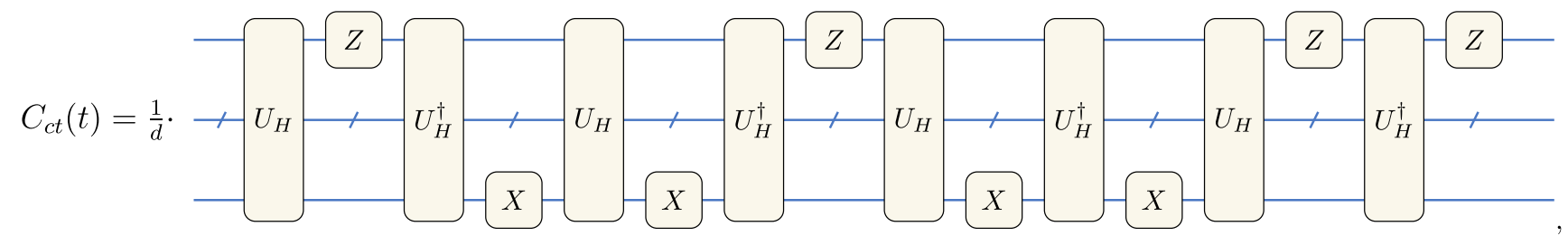

(E10)

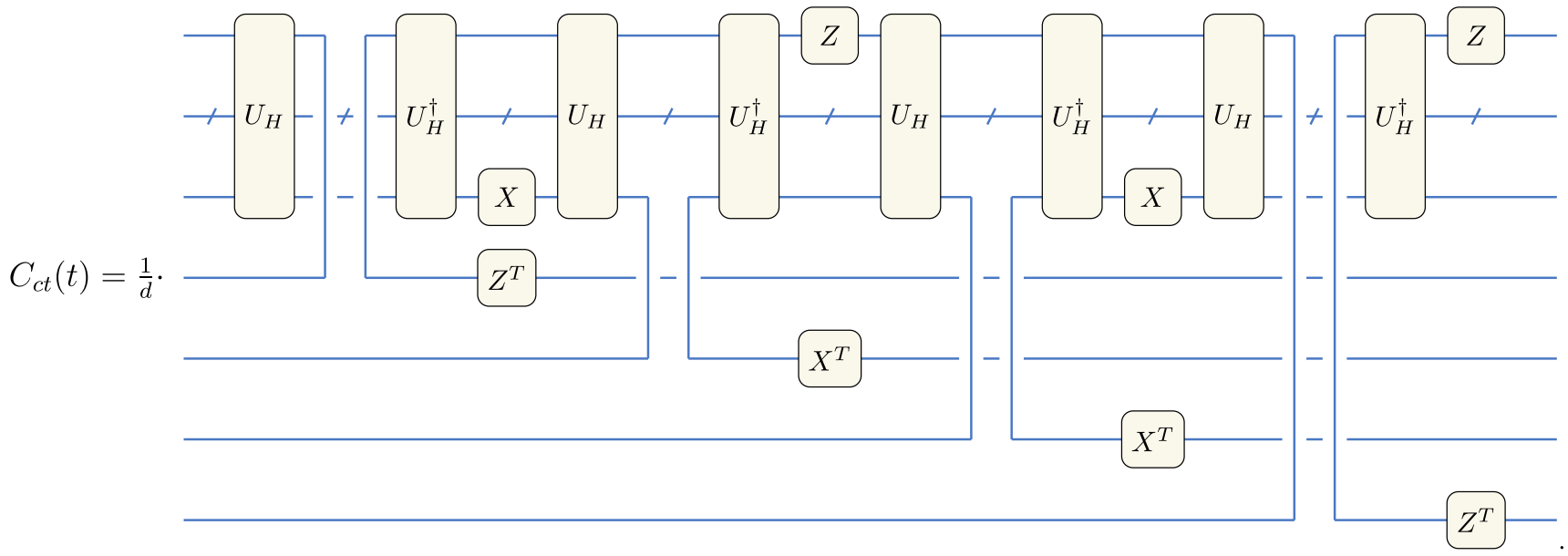

(E11)

Retrieve the definition of $\rho_{H, N, a_{1}}$ from Sec. IIID. Define $\rho_{1, a_{1}}$ as the state in which system qubit 1 forms a Bell state with one ancillary qubit, while the remaining qubits are in the maximally mixed state. Define the time-evolved state

$$
\rho_{H, 1, a_{1}}=\left(U_{H} \otimes I_{a_{1}}\right) \rho_{1, a_{1}}\left(U_{H}^{\dagger} \otimes I_{a_{1}}\right)
$$

The correlator from Eq. (E11) can be written as

$$
C_{c t}(t)=d^{3} \operatorname{Tr}\left\{\left(\rho_{H, 1, a_{1}} \otimes \rho_{H, N, a_{1}} \otimes \rho_{H, N, a_{1}} \otimes \rho_{H, 1, a_{1}}\right) O_{c t}\right\},
$$

where

$$
O_{c t}=\left(X_{N} Z_{a_{1}}^{T} \otimes Z_{1} X_{a_{1}}^{T} \otimes X_{N} X_{a_{1}}^{T} \otimes Z_{1} Z_{a_{1}}^{T}\right) \prod_{l=1}^{N} T_{(l, N+1+l, 2(N+1)+l, 3(N+1)+l)} .
$$

Prepare a shadow of size $K \geqslant 2$ for $\rho_{H, 1, a_{1}}$ and $\rho_{H, N, a_{1}}$. These two shadows can be used to compute the estimator

$$
\hat{C}_{c t}(t)=\frac{d^{3}}{4}\left(\begin{array}{c}
K \\
2
\end{array}\right)^{-2} \sum_{\substack{i_{1} \neq i_{4} \\
i_{2} \neq i_{3}}}^{K} \operatorname{Tr}\left\{\left(\hat{\rho}_{H, 1, a_{1}}^{\left(i_{1}\right)} \otimes \hat{\rho}_{H, N, a_{1}}^{\left(i_{2}\right)} \otimes \hat{\rho}_{H, N, a_{1}}^{\left(i_{3}\right)} \otimes \hat{\rho}_{H, 1, a_{1}}^{\left(i_{4}\right)}\right) O_{c t}\right\} .
$$


The commutator protocol to estimate $C_{c t}(t)$ is

(1) Prepare $N$ system qubits and 1 ancillary qubit. Create a Bell state between system qubit $N$ and the ancillary qubit. Prepare the remaining system qubits in the maximally mixed state.

(2) Evolve the system qubits with $U_{H}(t)$

(3) Construct a shadow of size $K \geqslant 2$ for the state.

(4) Prepare $N$ system qubits and 1 ancillary qubit. Create a Bell state between system qubit 1 and the ancillary qubit. Prepare the remaining system qubits in the maximally mixed state.

(5) Evolve the system qubits with $U_{H}(t)$.

(6) Construct a shadow of size $K \geqslant 2$ for the state.

(7) Use both shadows to compute $\hat{C}_{c t}(t)$.

This requires the preparation of two different states. However, by introducing two ancillary qubits and generating two
Bell states, this protocol can be implemented through the preparation of just one state.

\section{APPENDIX F: PROOFS}

\section{Proof of Lemma 1}

Proof. We begin the proof by first taking $O_{2}=T_{(1,2)}$, and later show that it can be used to bound the original case $O_{2}=T_{(1,2)} W^{\otimes 2}$. The swap operator $T_{(1,2)}$ on $\mathcal{H}_{d}^{\otimes 2}$ can be decomposed in the Pauli basis

$$
T_{(1,2)}=\frac{1}{d} \sum_{i} P_{i} \otimes P_{i},
$$

where $P_{i}$ is a Pauli operator and the sum is carried out over the $\mathrm{N}$-qubit Pauli group. Following the variance analysis for the shadow norm (see Eq. (S53) in Ref. [35]), the variance can be upper bounded by

$$
\begin{aligned}
& \max _{\sigma=\rho^{\otimes 2}} \mathbb{E}_{U \sim \mathrm{Cl}(2)^{\otimes 2 N}} \sum_{b \in\{0,1\}^{2 N}}\left\langle b\left|U \sigma U^{\dagger}\right| b\right\rangle\left\langle b\left|U\left(\mathcal{D}_{\frac{1}{3}}^{-1}\right)^{\otimes 2 N}\left(T_{(1,2)}\right) U^{\dagger}\right| b\right\rangle^{2} \\
& =\max _{\sigma=\rho^{\otimes 2}} \frac{1}{d^{2}} \sum_{i, j} \mathbb{E}_{U \sim \mathrm{Cl}(2)^{\otimes 2 N}} \sum_{b \in\{0,1\}^{2 N}}\left\langle b\left|U \sigma U^{\dagger}\right| b\right\rangle\left\langle b\left|U\left(\mathcal{D}_{\frac{1}{3}}^{-1}\right)^{\otimes 2 N}\left(P_{i}^{\otimes 2}\right) U^{\dagger}\right| b\right\rangle\left\langle b\left|U\left(\mathcal{D}_{\frac{1}{3}}^{-1}\right)^{\otimes 2 N}\left(P_{j}^{\otimes 2}\right) U^{\dagger}\right| b\right\rangle \\
& =\max _{\sigma=\rho^{\otimes 2}} \frac{1}{d^{2}} \sum_{i, j} f(i, j)^{2} \operatorname{Tr}\left\{\sigma P_{i}^{\otimes 2} P_{j}^{\otimes 2}\right\} \\
& =\max _{\rho} \frac{1}{d^{2}} \sum_{i, j} f(i, j)^{2} \operatorname{Tr}\left\{\rho P_{i} P_{j}\right\}^{2},
\end{aligned}
$$

where in the second line we insert the decomposition of the swap operator, and $f(i, j)$ is a function for two Pauli operators defined in Lemma 4 in Ref. [35]. $f(i, j)=0$ if there exists a qubit index $k$ such that the $k$ th qubit operators $P_{i}^{k} \neq P_{j}^{k}$ and $P_{i}^{k}, P_{j}^{k} \neq I$. Otherwise, $f(i, j)=3^{s}$, where $\mathrm{s}$ is the number of nonidentity Pauli indices that match. We remark that, differently from the shadow norm, our maximization is on the subset of the states in $\mathcal{H}_{d}^{\otimes 2}$ with a two-copy structure, i.e., states of the form $\sigma=\rho^{\otimes 2}$. This tensor structure leads to the tighter bound on the variance.

We introduce three functions denoted by $a, b, c$ for the two $N$-qubit Pauli operators $P_{i}, P_{j}$ indexed by $i, j . a(i, j)$ denotes the qubit positions for which $P_{i}, P_{j}$ share the same singlequbit Pauli operator; $b(i, j)$ denotes the positions where $P_{i}$ has a single-qubit Pauli operator and $P_{j}$ has $I$ (or vice versa); $c(i, j)$ denotes the positions where $P_{i}, P_{j}$ both have $I$. For example, for a six-qubit system with $P_{i}=X_{1} Y_{2} X_{3} I_{4} I_{5} I_{6}$ and $P_{j}=X_{1} Y_{2} I_{3} Z_{4} I_{5} I_{6}$, the functions are $a(i, j)=\{1,2\}, b(i, j)=$ $\{3,4\}$ and $c(i, j)=\{5,6\}$. We use $|a(i, j)|$ to denote the number of elements in $a(i, j)$ and omit the $i, j$ indices when there is no ambiguity. $P_{i}^{a}$ is the $|a|$-qubit operator restricted on subsystem $a$ from $P_{i}$.

It is not necessary to consider the $P_{i}, P_{j}$ pair with different Pauli operators acting on the same qubit, since they return $f(i, j)=0$. As a result, the summation in Eq. (F2) can be transformed to the summation on all possible $a, b, c$ with $|a|+|b|+|c|=n$. We use $(i, j) \vdash(a, b, c)$ to denote that $P_{i}, P_{j}$ satisfy the operator constraints on the given $a, b, c$ subsystems. We write

$$
\begin{aligned}
& \sum_{i, j} f(i, j)^{2} \operatorname{Tr}\left\{\rho P_{i} P_{j}\right\}^{2} \\
& =\sum_{a, b, c} 3^{2|a|} \sum_{(i, j) \vdash(a, b, c)} \operatorname{Tr}\left\{\rho I_{a} \otimes P_{i}^{b} P_{j}^{b} \otimes I_{c}\right\}^{2} \\
& =\sum_{a, b, c} 3^{2|a|} \sum_{(i, j) \vdash(a, b, c)} \operatorname{Tr}\left\{\rho_{b} P_{i}^{b} P_{j}^{b}\right\}^{2} .
\end{aligned}
$$

Here $3^{2|a|}$ accounts for the function $f(i, j)^{2}$, and we use the fact that the qubit Pauli operators of $P_{i}, P_{j}$ are the same in $a$ and they both have $I$ on $c$.

There are $3^{|a|}$ possible choices of Paulis $\{X, Y, Z\}^{|a|}$ on subsystem $a . P_{b}^{i} P_{b}^{j}$ can take all Pauli operators $\{X, Y, Z\}^{|b|}$ on $b$ by the property of the $b$ function. For each choice of operators on $a$, we can sum the result of all of these possible Paulis on $b$. One thus has

$$
\begin{aligned}
\sum_{(i, j) \vdash(a, b, c)} \operatorname{Tr}\left\{\rho_{b} P_{b}^{i} P_{b}^{j}\right\}^{2} & =3^{|a|} 2^{|b|} \sum_{\tilde{P}_{b} \in\{X, Y, Z\}^{|b|}} \operatorname{Tr}\left\{\rho_{b} \tilde{P}_{b}\right\}^{2} \\
& \leqslant 3^{|a|} 2^{|b|} \sum_{\tilde{P}_{b} \in\{\mathbb{I}, X, Y, Z\}^{|b|}} \operatorname{Tr}\left\{\rho_{b} \tilde{P}_{b}\right\}^{2} \\
& =3^{|a|} 2^{|b|} 2^{|b|} \operatorname{Tr}\left\{\rho_{b}^{2}\right\} \\
& \leqslant 3^{|a|} 2^{2|b|} .
\end{aligned}
$$


The $2^{|b|}$ in the first line is due to the two possibilities for $P_{i}$ or $P_{j}$ taking a Pauli or the identity on the qubit in $b$. The last inequality is due to the purity $\operatorname{Tr}\left\{\rho_{b}^{2}\right\} \leqslant 1$. Inserting Eq. (F4) into Eq. (F3), we get the upper bound for the variance:

$$
\begin{aligned}
& \frac{1}{d^{2}} \sum_{i, j} f(i, j)^{2} \operatorname{Tr}\left\{\rho P_{i} P_{j}\right\}^{2} \\
& =\frac{1}{d^{2}} \sum_{a, b, c} 3^{2|a|} \sum_{(i, j) \vdash(a, b, c)} \operatorname{Tr}\left\{\rho_{b} P_{b}^{i} P_{b}^{j}\right\}^{2} \\
& \leqslant \frac{1}{d^{2}} \sum_{|a|+|b|+|c|=N}\left(\begin{array}{c}
N \\
|a|
\end{array}\right)\left(\begin{array}{c}
N-|a| \\
|b|
\end{array}\right)\left(\begin{array}{c}
N-|a|-|b| \\
|c|
\end{array}\right) 3^{3|a|} 2^{2|b|} \\
& =\frac{\left(3^{3}+2^{2}+1\right)^{N}}{d^{2}}=d^{3} .
\end{aligned}
$$

For the case $O_{2}=T_{(1,2)} W^{\otimes 2}$ with $W$ a Pauli operator, as in Eq. (F3) one has that the variance is upper bounded by

$$
\begin{aligned}
\operatorname{Var} & {\left[\operatorname{Tr}\left\{O_{2} \hat{\rho} \otimes \hat{\rho}^{\prime}\right\}\right] } \\
& \leqslant \max _{\sigma=\rho^{\otimes 2}} \frac{1}{d^{2}} \sum_{i, j} f(i, j)^{2} \operatorname{Tr}\left\{\sigma P_{i}^{\otimes 2} W^{\otimes 2} P_{j}^{\otimes 2} W^{\otimes 2}\right\} \\
& =\max _{\rho} \frac{1}{d^{2}} \sum_{i, j} f(i, j)^{2} \operatorname{Tr}\left\{\rho P_{i} W P_{j} W\right\}^{2} \\
& =\max _{\rho} \frac{1}{d^{2}} \sum_{i, j} f(i, j)^{2} \operatorname{Tr}\left\{\rho P_{i} P_{j}\right\}^{2} \leqslant d^{3},
\end{aligned}
$$

where we use the fact $\operatorname{Tr}\left\{\rho P_{i} W P_{j} W\right\}^{2}=\operatorname{Tr}\left\{\rho P_{i} P_{j}\right\}^{2}$ for any Pauli operator.

\section{Proof of Proposition 2}

Proof. We rewrite the variance of $\hat{L}_{8}$ from main text here:

$$
\begin{aligned}
\operatorname{Var}\left(\hat{L}_{8}\right) & =\left[d^{3}\left(\begin{array}{l}
K \\
4
\end{array}\right)^{-1}\right]^{2} \operatorname{Var}\left(\sum_{\vec{i}} \hat{D}_{8}(\vec{i})\right) \\
& =d^{6}\left(\begin{array}{c}
K \\
4
\end{array}\right)^{-2} \sum_{\vec{i}, \vec{j}}\left\{\mathbb{E}\left[\hat{D}_{8}(\vec{i}) \hat{D}_{8}(\vec{j})\right]-D_{8}^{2}\right\} \\
& =d^{6}\left(\begin{array}{c}
K \\
4
\end{array}\right)^{-2} \sum_{\vec{i}, \vec{j}}\left[V_{8}(\vec{i}, \vec{j})-D_{8}^{2}\right],
\end{aligned}
$$

where the summation over $\vec{i}$ labels the summation over $i_{1}<$ $i_{2}<i_{3}<i_{4}$, and similarly for $\vec{j}$.

Similarly to the analysis of $\hat{C}_{4}$ in Sec. IV A, the variance $\operatorname{Var}\left(\hat{L}_{8}\right)$ depends on the coincidences of the indices. For simplicity of notation, we use $T_{t}$ to denote the shift operator $T_{(1,2, \ldots, t)}$ in the following discussion.

(i) No coincidence: $V_{8}=D_{8}^{2}$, since the snapshots are independent and one can evaluate the expectation value separately.

(ii) One coincidence: there are a total of $\left(\begin{array}{c}M \\ 1\end{array}\right)\left(\begin{array}{c}M-1 \\ 6\end{array}\right)\left(\begin{array}{l}6 \\ 3\end{array}\right)$ such terms. We have

$$
\begin{aligned}
V_{8} & =\mathbb{E}\left[\operatorname{Tr}\left\{\Theta_{4}\left(T_{4}\right) W^{\otimes 4} \rho_{V}^{\otimes 3} \otimes \hat{\rho}_{V}\right\}^{2}\right] \\
& =\mathbb{E}\left[\operatorname{Tr}\left\{T_{4} W^{\otimes 4} \rho_{V}^{\otimes 3} \otimes \hat{\rho}_{V}\right\}^{2}\right] \\
& =\mathbb{E}\left[\operatorname{Tr}\left\{W \rho_{V} W \rho_{V} W \rho_{V} W \hat{\rho}_{V}\right\}^{2}\right] .
\end{aligned}
$$

The second line is due to the six different permutation orders returning the same result. We can take $O_{1}$ as $O_{1}=$ $W \rho_{V} W \rho_{V} W \rho_{V} W$, and $\mathbb{E}\left[\operatorname{Tr}\left\{O_{1} \hat{\rho}_{V}\right\}\right]=D_{8}$. In this way, $V_{8}-$ $D_{8}^{2}=\operatorname{Var}\left[\operatorname{Tr}\left\{O_{1} \hat{\rho}_{V}\right\}\right]$, and one can analyze the variance based on Fact 1 with $\operatorname{Tr}\left\{O_{1}^{2}\right\}=\frac{4}{d^{2}} D_{8}$.

(iii) Two coincidences: there are a total of $\left(\begin{array}{c}M \\ 2\end{array}\right)\left(\begin{array}{c}M-2 \\ 4\end{array}\right)\left(\begin{array}{l}4 \\ 2\end{array}\right)$ such terms. Due to the redundancy of the twirling channel $\Theta_{4}$, we only need to consider the average on the following three orders

$$
\begin{aligned}
V_{8} & =\mathbb{E}\left[\left(\frac{1}{3} \operatorname{Tr}\left\{T_{4} W^{\otimes 4} \rho_{V}^{\otimes 2} \otimes \hat{\rho}_{V}^{(1)} \otimes \hat{\rho}_{V}^{(2)}\right\}+\frac{1}{3} \operatorname{Tr}\left\{T_{4} W^{\otimes 4} \rho_{V}^{\otimes 2} \otimes \hat{\rho}_{V}^{(2)} \otimes \hat{\rho}_{V}^{(1)}\right\}+\frac{1}{3} \operatorname{Tr}\left\{T_{4} W^{\otimes 4} \rho_{V} \otimes \hat{\rho}_{V}^{(1)} \otimes \rho_{V} \otimes \hat{\rho}_{V}^{(2)}\right\}\right)^{2}\right] \\
& =\mathbb{E}\left[\left(\frac{1}{3} \operatorname{Tr}\left\{T_{2} O^{\prime} \otimes W \hat{\rho}_{V}^{(1)} \otimes \hat{\rho}_{V}^{(2)}\right\}+\frac{1}{3} \operatorname{Tr}\left\{T_{2} W \otimes O^{\prime} \hat{\rho}_{V}^{(1)} \otimes \hat{\rho}_{V}^{(2)}\right\}+\frac{1}{3} \operatorname{Tr}\left\{T_{2}\left[W \rho_{V} W\right]^{\otimes 2} \hat{\rho}_{V}^{(1)} \otimes \hat{\rho}_{V}^{(2)}\right\}\right)^{2}\right],
\end{aligned}
$$

where $O^{\prime}=W \rho_{V} W \rho_{V} W$. We take the final observable $O_{2}=\frac{1}{3}\left(T_{2} O^{\prime} \otimes W+T_{2} W \otimes O^{\prime}+T_{2}\left[W \rho_{V} W\right]^{\otimes 2}\right)$ to analyze the variance based on Fact 1 with

$$
\begin{aligned}
\operatorname{Tr}\left\{O_{2}^{2}\right\}= & \frac{1}{9} \operatorname{Tr}\left\{\left(O^{\prime} \otimes W+W \otimes O^{\prime}+\left[W \rho_{V} W\right]^{\otimes 2}\right)^{2}\right\} \\
= & \frac{1}{9}\left(2 d \operatorname{Tr}\left\{O^{\prime 2}\right\}+2 \operatorname{Tr}\left\{O^{\prime} W\right\}^{2}+4 \operatorname{Tr}\left\{O^{\prime} W \rho_{V} W\right\}\right. \\
& \left.\times \operatorname{Tr}\left\{\rho_{V} W\right\}+\operatorname{Tr}\left\{\left(W \rho_{V} W\right)^{2}\right\}^{2}\right)
\end{aligned}
$$

$$
=\frac{1}{9}\left(\frac{8}{d} D_{4}+2 D_{4}^{2}+\frac{16}{d^{2}} D_{2}^{2}+\frac{4}{d^{2}}\right)
$$

with $D_{2}=\operatorname{Tr}\left\{W \rho_{V}\right\}$ and $D_{4}=\operatorname{Tr}\left\{W \rho_{V} W \rho_{V}\right\}=\left(C_{4}+1\right) / d$.

(iv) Three coincidences: there are a total of $\left(\begin{array}{c}M \\ 3\end{array}\right)\left(\begin{array}{c}M-3 \\ 2\end{array}\right)\left(\begin{array}{l}2 \\ 1\end{array}\right)$ such terms. Similarly to the two previous coincidence cases, one can get

$$
V_{8}=\mathbb{E}\left[\operatorname{Tr}\left\{O_{3} \hat{\rho}_{V}^{(1)} \otimes \hat{\rho}_{V}^{(2)} \otimes \hat{\rho}_{V}^{(3)}\right\}^{2}\right]
$$


where $O_{3}=\frac{1}{2}\left(T_{3}+T_{3}^{\dagger}\right) \Theta_{3}\left(O^{\prime \prime} \otimes W^{\otimes 2}\right)$, with $O^{\prime \prime}=W \rho_{V} W$. The variance can be bounded by Fact 1 with

$$
\begin{aligned}
\operatorname{Tr}\left\{O_{3}^{2}\right\} & =\operatorname{Tr}\left\{\frac{1}{4}\left(2 \mathbb{I}+T_{3}^{2}+T_{3}^{\dagger 2}\right) \Theta_{3}\left(O^{\prime \prime} \otimes \mathbb{I}^{\otimes 2}\right)^{2}\right\} \\
& =\frac{1}{2} \operatorname{Tr}\left\{\left(\mathbb{I}+T_{3}\right) \Theta_{3}\left(O^{\prime \prime} \otimes \mathbb{I}^{\otimes 2}\right)^{2}\right\} \\
& =\frac{1}{6}\left[\operatorname{Tr}\left\{O^{\prime \prime 2}\right\} d^{2}+2 \operatorname{Tr}\left\{O^{\prime \prime} W\right\}^{2} d\right]+\frac{1}{2} \operatorname{Tr}\left\{O^{\prime \prime 2}\right\} \\
& =\frac{d\left(1+D_{2}^{2}\right)}{3}+\frac{1}{d},
\end{aligned}
$$

where we use $\operatorname{Tr}\left\{O^{\prime \prime 2}\right\}=\frac{2}{d}$.

$$
\begin{aligned}
& \operatorname{Var}\left(\hat{L}_{8}\right)=d^{6}\left(\begin{array}{c}
K \\
4
\end{array}\right)^{-2} \sum_{\vec{i}, \vec{j}}\left[V_{8}(\vec{i}, \vec{j})-D_{8}^{2}\right] \\
& =d^{6}\left(\begin{array}{l}
K \\
4
\end{array}\right)^{-2} \sum_{c=1}^{4} N_{c} \operatorname{Var}\left(\hat{O}_{c}\right) \\
& \leqslant d^{6}\left(\begin{array}{l}
K \\
4
\end{array}\right)^{-1} \sum_{c=1}^{4}\left(\begin{array}{l}
4 \\
c
\end{array}\right)\left(\begin{array}{l}
K-4 \\
4-c
\end{array}\right) \operatorname{Tr}\left(O_{c}^{2}\right) d^{c} \\
& =\frac{24 d^{6}}{K(K-1)(K-2)(K-3)}\left\{4 \times \frac{1}{6}(K-4)(K-5)(K-6) \frac{4}{d^{2}} D_{8} d+6 / 2(K-4)(K-5)\right. \\
& \left.\times \frac{1}{9}\left(\frac{8}{d} D_{4}+2 D_{4}^{2}+\frac{16}{d^{2}} D_{2}^{2}+\frac{4}{d^{2}}\right) d^{2}+4(K-4)\left[\frac{d\left(1+D_{2}^{2}\right)}{3}+\frac{1}{d}\right] d^{3}+\frac{d^{4}+5 d^{2}}{6} d^{4}\right\} \\
& \leqslant \frac{64 d^{5} D_{8}}{K}+\frac{16\left(4 d D_{4}+d^{2} D_{4}^{2}+8 D_{2}^{2}+2\right)}{K^{2}}+\frac{32\left[d^{10}\left(1+D_{2}^{2}\right)+3 d^{8}\right]}{K^{3}}+\frac{4\left(d^{14}+5 d^{6}\right)}{K^{4}} \text {. }
\end{aligned}
$$

(v) Four coincidences: this corresponds to $\vec{i}=\vec{j}$, with $\left(\begin{array}{c}M \\ 4\end{array}\right)$ such terms:

$$
V_{8}=\mathbb{E}\left[\operatorname{Tr}\left\{O_{4} \hat{\rho}_{V}^{(1)} \otimes \hat{\rho}_{V}^{(2)} \otimes \hat{\rho}_{V}^{(3)} \otimes \hat{\rho}_{V}^{(4)}\right\}^{2}\right],
$$

where $O_{4}=\Theta_{4}\left(T_{4}\right) W^{\otimes 4}$ for the four-copy state, and

$$
\begin{aligned}
\operatorname{Tr}\left\{O_{4}^{2}\right\} & =\operatorname{Tr}\left\{\Theta_{4}\left(T_{4}\right) \Theta_{4}\left(T_{4}\right)\right\} \\
& =\operatorname{Tr}\left\{\Theta_{4}\left(T_{4}\right) T_{4}\right\} \\
& =\frac{d^{4}+5 d^{2}}{6} .
\end{aligned}
$$

For simplicity, denote the number of terms in each case as $N_{c}=\left(\begin{array}{c}K \\ c\end{array}\right)\left(\begin{array}{c}K-c \\ 8-2 c\end{array}\right)\left(\begin{array}{c}8-2 c \\ 4-2 c\end{array}\right)=\left(\begin{array}{c}K \\ 4\end{array}\right)\left(\begin{array}{l}4 \\ c\end{array}\right)\left(\begin{array}{c}K-4 \\ 4-c\end{array}\right)$ for $c=1,2,3,4$, and denote the variance of $O_{c}$ on $c$ snapshots as $\operatorname{Var}\left(\hat{O}_{c}\right)$. Combining the above cases, one has
Focusing on the early-time behavior where $d^{3} D_{8} \sim 8, d D_{4} \sim$ 2 and also using $\left|D_{2}\right| \leqslant 1$, the variance satisfies

$$
\begin{aligned}
\operatorname{Var}\left(\hat{L}_{8}\right) \leqslant & \frac{512 d^{2}}{K}+\frac{352}{K^{2}}+\frac{32\left(2 d^{10}+3 d^{8}\right)}{K^{3}} \\
& +\frac{4\left(d^{14}+5 d^{6}\right)}{K^{4}} .
\end{aligned}
$$

It is clear that, in the large $d$ limit, the final term is dominating and the variance scales as $O\left(d^{14} / K^{4}\right)$. To suppress the error to $\epsilon$, one needs $K=O\left(d^{3.5} / \sqrt{\epsilon}\right)$. If one naively uses quantum state tomography to reconstruct $\rho_{V}$ and calculate $L_{8}=d^{3} D_{8}=d^{3} \operatorname{Tr}\left\{\left(W \rho_{V}\right)^{4}\right\}$ within an error $\epsilon$, one needs the precision of tomography to be about $\epsilon^{\prime}=\epsilon / d^{3}$. Even with the optimistic estimation obtained by making the trace distance comparable to the infidelity, the necessary number of measurements in quantum tomography scales as $K=\Omega\left(d^{2} / \epsilon^{\prime}\right)=$ $\Omega\left(d^{5} / \epsilon\right)$. Furthermore, if one is restricted to independent measurements on a single-copy of $\rho_{V}$ (like in the classical shadow protocol here), the scaling gets worse.
[1] R. J. Lewis-Swan, A. Safavi-Naini, A. M. Kaufman, and A. M. Rey, Dynamics of quantum information, Nat. Rev. Phys. 1, 627 (2019).

[2] S. H. Shenker and D. Stanford, Black holes and the butterfly effect, J. High Energy Phys. 03 (2014) 067.

[3] P. Hayden and J. Preskill, Black holes as mirrors: quantum information in random subsystems, J. High Energy Phys. 09 (2007) 120.

[4] Y. Sekino and L. Susskind, Fast scramblers, J. High Energy Phys. 10 (2008) 065.
[5] J. Maldacena, S. H. Shenker, and D. Stanford, A bound on chaos, J. High Energy Phys. 08 (2016) 106.

[6] S. Sachdev and J. Ye, Gapless Spin-Fluid Ground State in a Random Quantum Heisenberg Magnet, Phys. Rev. Lett. 70, 3339 (1993).

[7] A. Kitaev, A simple model of quantum holography, https:// online.kitp.ucsb.edu/online/entangled15/.

[8] D. A. Roberts and D. Stanford, Diagnosing Chaos Using FourPoint Functions in Two-Dimensional Conformal Field Theory, Phys. Rev. Lett. 115, 131603 (2015). 
[9] B. Swingle, G. Bentsen, M. Schleier-Smith, and P. Hayden, Measuring the scrambling of quantum information, Phys. Rev. A 94, 040302(R) (2016).

[10] D. Chowdhury and B. Swingle, Onset of many-body chaos in the $O(N)$ model, Phys. Rev. D 96, 065005 (2017).

[11] M. C. Bañuls, J. I. Cirac, and M. B. Hastings, Strong and Weak Thermalization of Infinite Nonintegrable Quantum Systems, Phys. Rev. Lett. 106, 050405 (2011).

[12] R. H. Dicke, Coherence in spontaneous radiation processes, Phys. Rev. 93, 99 (1954).

[13] Y. Alavirad and A. Lavasani, Scrambling in the Dicke model, Phys. Rev. A 99, 043602 (2019).

[14] R. J. Lewis-Swan, A. Safavi-Naini, J. J. Bollinger, and A. M. Rey, Unifying scrambling, thermalization and entanglement through measurement of fidelity out-of-time-order correlators in the Dicke model, Nat. Commun. 10, 1581 (2019).

[15] G. Bentsen, T. Hashizume, A. S. Buyskikh, E. J. Davis, A. J. Daley, S. S. Gubser, and M. Schleier-Smith, Treelike Interactions and Fast Scrambling with Cold Atoms, Phys. Rev. Lett. 123, 130601 (2019).

[16] R. Belyansky, P. Bienias, Y. A. Kharkov, A. V. Gorshkov, and B. Swingle, Minimal Model for Fast Scrambling, Phys. Rev. Lett. 125, 130601 (2020).

[17] B. Swingle and D. Chowdhury, Slow scrambling in disordered quantum systems, Phys. Rev. B 95, 060201(R) (2017).

[18] Y. Huang, Y.-L. Zhang, and X. Chen, Out-of-time-ordered correlators in many-body localized systems, Ann. Phys. 529, 1600318 (2016).

[19] R. Fan, P. Zhang, H. Shen, and H. Zhai, Out-of-time-order correlation for many-body localization, Sci. Bull. 62, 707 (2017).

[20] Y. Chen, Universal logarithmic scrambling in many body localization, arXiv:1608.02765.

[21] X. Chen, T. Zhou, D. A. Huse, and E. Fradkin, Out-of-timeorder correlations in many-body localized and thermal phases, Ann. Phys. (NY) 529, 1600332 (2016).

[22] R.-Q. He and Z.-Y. Lu, Characterizing many-body localization by out-of-time-ordered correlation, Phys. Rev. B 95, 054201 (2017).

[23] M. Gärttner, J. G. Bohnet, A. Safavi-Naini, M. L. Wall, J. J. Bollinger, and A. M. Rey, Measuring out-of-time-order correlations and multiple quantum spectra in a trapped-ion quantum magnet, Nat. Phys. 13, 781 (2017).

[24] K. X. Wei, C. Ramanathan, and P. Cappellaro, Exploring Localization in Nuclear Spin Chains, Phys. Rev. Lett. 120, 070501 (2018).

[25] J. Li, R. Fan, H. Wang, B. Ye, B. Zeng, H. Zhai, X. Peng, and J. Du, Measuring Out-of-Time-Order Correlators on a Nuclear Magnetic Resonance Quantum Simulator, Phys. Rev. X 7, 031011 (2017).

[26] X. Mi, P. Roushan, C. Quintana, S. Mandra, J. Marshall, C. Neill, F. Arute, K. Arya, J. Atalaya, R. Babbush, J. C. Bardin, R. Barends, A. Bengtsson, S. Boixo, A. Bourassa, M. Broughton, B. B. Buckley, D. A. Buell, B. Burkett, N. Bushnell et al., Information scrambling in computationally complex quantum circuits, arXiv:2101.08870.

[27] B. Yoshida and N. Y. Yao, Disentangling Scrambling and Decoherence via Quantum Teleportation, Phys. Rev. X 9, 011006 (2019).
[28] K. A. Landsman, C. Figgatt, T. Schuster, N. M. Linke, B. Yoshida, N. Y. Yao, and C. Monroe, Verified quantum information scrambling, Nature (London) 567, 61 (2019).

[29] M. S. Blok, V. V. Ramasesh, T. Schuster, K. O’Brien, J. M Kreikebaum, D. Dahlen, A. Morvan, B. Yoshida, N. Y. Yao, and I. Siddiqi, Quantum Information Scrambling on a Superconducting Qutrit Processor, Phys. Rev. X 11, 021010 (2021).

[30] B. Vermersch, A. Elben, L. M. Sieberer, N. Y. Yao, and P. Zoller, Probing Scrambling Using Statistical Correlations between Randomized Measurements, Phys. Rev. X 9, 021061 (2019).

[31] M. K. Joshi, A. Elben, B. Vermersch, T. Brydges, C. Maier, P. Zoller, R. Blatt, and C. F. Roos, Quantum Information Scrambling in a Trapped-ion Quantum Simulator with Tunable Range Interactions, Phys. Rev. Lett. 124, 240505 (2020).

[32] S. H. Shenker and D. Stanford, Multiple shocks, J. High Energy Phys. 12 (2014) 046.

[33] D. A. Roberts and B. Yoshida, Chaos and complexity by design, J. High Energy Phys. 04 (2017) 121.

[34] S. Aaronson, Shadow tomography of quantum states, arXiv:1711.01053.

[35] H.-Y. Huang, R. Kueng, and J. Preskill, Predicting many properties of a quantum system from very few measurements, Nat. Phys. 16, 1050 (2020).

[36] A. K. Ekert, C. M. Alves, D. K. L. Oi, M. Horodecki, P. Horodecki, and L. C. Kwek, Direct Estimations of Linear and Nonlinear Functionals of a Quantum State, Phys. Rev. Lett. 88, 217901 (2002).

[37] A. J. Daley, H. Pichler, J. Schachenmayer, and P. Zoller, Measuring Entanglement Growth in Quench Dynamics of Bosons in an Optical Lattice, Phys. Rev. Lett. 109, 020505 (2012).

[38] D. A. Abanin and E. Demler, Measuring Entanglement Entropy of a Generic Many-Body System with a Quantum Switch, Phys. Rev. Lett. 109, 020504 (2012).

[39] R. Islam, R. Ma, P. M. Preiss, M. Eric Tai, A. Lukin, M. Rispoli, and M. Greiner, Measuring entanglement entropy in a quantum many-body system, Nature (London) 528, 77 (2015).

[40] A. M. Kaufman, M. E. Tai, A. Lukin, M. Rispoli, R. Schittko, P. M. Preiss, and M. Greiner, Quantum thermalization through entanglement in an isolated many-body system, Science $\mathbf{3 5 3}$, 794 (2016).

[41] N. Y. Yao, F. Grusdt, B. Swingle, M. D. Lukin, D. M. StamperKurn, J. E. Moore, and E. A. Demler, Interferometric approach to probing fast scrambling, arXiv:1607.01801.

[42] A. Elben, R. Kueng, H.-Y. R. Huang, R. van Bijnen, C. Kokail, M. Dalmonte, P. Calabrese, B. Kraus, J. Preskill, P. Zoller, and B. Vermersch, Mixed-State Entanglement from Local Randomized Measurements, Phys. Rev. Lett. 125, 200501 (2020).

[43] Z. P. Karkuszewski, C. Jarzynski, and W. H. Zurek, Quantum Chaotic Environments, the Butterfly Effect, and Decoherence, Phys. Rev. Lett. 89, 170405 (2002).

[44] E. H. Lieb and D. W. Robinson, The finite group velocity of quantum spin systems, Commun. Math. Phys. 28, 251 (1972).

[45] R. Bhattacharya, D. P. Jatkar, and A. Kundu, Chaotic correlation functions with complex fermions, arXiv:1810.13217.

[46] J. Iaconis, Quantum State Complexity in Computationally Tractable Quantum Circuits, PRX Quantum 2, 010329 (2021). 
[47] S. J. van Enk and C. W. J. Beenakker, Measuring $\operatorname{Tr} \rho^{n}$ on Single Copies of $\rho$ Using Random Measurements, Phys. Rev. Lett. 108, 110503 (2012)

[48] A. Elben, B. Vermersch, C. F. Roos, and P. Zoller, Statistical correlations between locally randomized measurements: A toolbox for probing entanglement in many-body quantum states, Phys. Rev. A 99, 052323 (2019).

[49] A. Elben, J. Yu, G. Zhu, M. Hafezi, F. Pollmann, P. Zoller, and B. Vermersch, Many-body topological invariants from randomized measurements in synthetic quantum matter, Sci. Adv. 6, eaaz3666 (2020).

[50] Z.-P. Cian, H. Dehghani, A. Elben, B. Vermersch, G. Zhu, M. Barkeshli, P. Zoller, and M. Hafezi, Many-Body Chern Number from Statistical Correlations of Randomized Measurements, Phys. Rev. Lett. 126, 050501 (2021).

[51] A. Elben, B. Vermersch, M. Dalmonte, J. I. Cirac, and P. Zoller, Rényi Entropies from Random Quenches in Atomic Hubbard and Spin Models, Phys. Rev. Lett. 120, 050406 (2018).

[52] T. Brydges, A. Elben, P. Jurcevic, B. Vermersch, C. Maier, B. P. Lanyon, P. Zoller, R. Blatt, and C. F. Roos, Probing Rényi entanglement entropy via randomized measurements, Science 364, 260 (2019).

[53] Y. Zhou, P. Zeng, and Z. Liu, Single-Copies Estimation of Entanglement Negativity, Phys. Rev. Lett. 125, 200502 (2020).

[54] A. Elben, B. Vermersch, R. van Bijnen, C. Kokail, T. Brydges, C. Maier, M. K. Joshi, R. Blatt, C. F. Roos, and P. Zoller, CrossPlatform Verification of Intermediate Scale Quantum Devices, Phys. Rev. Lett. 124, 010504 (2020).

[55] W.-H. Zhang, C. Zhang, Z. Chen, X.-X. Peng, X.-Y. Xu, P. Yin, S. Yu, X.-J. Ye, Y.-J. Han, J.-S. Xu, G. Chen, C.-F. Li, and G.-C. Guo, Experimental Optimal Verification of Entangled States Using Local Measurements, Phys. Rev. Lett. 125, 030506 (2020).

[56] M. C. Tran, B. Dakić, F. Arnault, W. Laskowski, and T. Paterek, Quantum entanglement from random measurements, Phys. Rev. A 92, 050301(R) (2015).

[57] M. C. Tran, B. Dakić, W. Laskowski, and T. Paterek, Correlations between outcomes of random measurements, Phys. Rev. A 94, 042302 (2016).

[58] A. Ketterer, N. Wyderka, and O. Gühne, Characterizing Multipartite Entanglement with Moments of Random Correlations, Phys. Rev. Lett. 122, 120505 (2019).

[59] A. Ketterer, N. Wyderka, and O. Gühne, Entanglement characterization using quantum designs, Quantum 4, 325 (2020).

[60] L. Knips, J. Dziewior, W. Kobus, W. Laskowski, T. Paterek, P. J. Shadbolt, H. Weinfurter, and J. D. A. Meinecke, Multipartite entanglement analysis from random correlations, npj Quantum Inf. 6, 51 (2020).

[61] D. Gross, K. Audenaert, and J. Eisert, Evenly distributed unitaries: On the structure of unitary designs, J. Math. Phys. 48, 052104 (2007).

[62] Z. Webb, The Clifford group forms a unitary 3-design, Quantum Inf. Comput. 16, 1379 (2016).

[63] H. Zhu, R. Kueng, M. Grassl, and D. Gross, The Clifford group fails gracefully to be a unitary 4-design, arXiv:1609.08172.

[64] R. Kueng and D. Gross, Qubit stabilizer states are complex projective 3-designs, arXiv:1510.02767.

[65] F. G. S. L. Brando, A. W. Harrow, and M. Horodecki, Local random quantum circuits are approximate polynomial-designs, Commun. Math. Phys. 346, 397 (2016).
[66] J. Haferkamp, F. Montealegre-Mora, M. Heinrich, J. Eisert, D. Gross, and I. Roth, Quantum homeopathy works: Efficient unitary designs with a system-size independent number of nonClifford gates, arXiv:2002.09524.

[67] P. Hosur, X.-L. Qi, D. A. Roberts, and B. Yoshida, Chaos in quantum channels, J. High Energy Phys. 02 (2016) 004.

[68] A. Jaffe, Z. Liu, and A. Wozniakowski, Holographic software for quantum networks, Sci. China Math. 61, 593 (2018).

[69] B. Collins and I. Nechita, Random quantum channels I: Graphical calculus and the Bell state phenomenon, Commun. Math. Phys. 297, 345 (2010).

[70] S. Kotz and N. Johnson, Breakthroughs in Statistics (U.S. GPO, Washington, DC, 1992), Vol. 3.

[71] H. Levine, A. Keesling, A. Omran, H. Bernien, S. Schwartz, A. S. Zibrov, M. Endres, M. Greiner, V. Vuletić, and M. D. Lukin, High-Fidelity Control and Entanglement of RydbergAtom Qubits, Phys. Rev. Lett. 121, 123603 (2018).

[72] B. Yang, H. Sun, C.-J. Huang, H.-Y. Wang, Y. Deng, H.-N. Dai, Z.-S. Yuan, and J.-W. Pan, Cooling and entangling ultracold atoms in optical lattices, Science 369, 550 (2020).

[73] J. Haah, A. W. Harrow, Z. Ji, X. Wu, and N. Yu, Sample-optimal tomography of quantum states, in Proceedings of the FortyEighth Annual ACM Symposium on Theory of Computing, STOC '16 (Association for Computing Machinery, New York, 2016), p. 913.

[74] N. Yu, Sample efficient tomography via Pauli measurements, arXiv:2009.04610.

[75] E. B. Rozenbaum, S. Ganeshan, and V. Galitski, Lyapunov Exponent and Out-of-Time-Ordered Correlator's Growth Rate in a Chaotic System, Phys. Rev. Lett. 118, 086801 (2017).

[76] C.-J. Lin and O. I. Motrunich, Out-of-time-ordered correlators in a quantum Ising chain, Phys. Rev. B 97, 144304 (2018).

[77] S. Xu and B. Swingle, Accessing scrambling using matrix product operators, Nat. Phys. 16, 199 (2019).

[78] S. Chen, W. Yu, P. Zeng, and S. T. Flammia, Robust shadow estimation, arXiv:2011.09636.

[79] D. E. Koh and S. Grewal, Classical shadows with noise, arXiv:2011.11580.

[80] B. Swingle and N. Yunger Halpern, Resilience of scrambling measurements, Phys. Rev. A 97, 062113 (2018).

[81] Y.-L. Zhang, Y. Huang, and X. Chen, Information scrambling in chaotic systems with dissipation, Phys. Rev. B 99, 014303 (2019).

[82] J. Kudler-Flam, M. Nozaki, S. Ryu, and M. T. Tan, Quantum vs. classical information: operator negativity as a probe of scrambling, J. High Energy Phys. 01 (2020) 031.

[83] D. A. Roberts, D. Stanford, and A. Streicher, Operator growth in the SYK model, J. High Energy Phys. 06 (2018) 122.

[84] X.-L. Qi, E. J. Davis, A. Periwal, and M. Schleier-Smith, Measuring operator size growth in quantum quench experiments, arXiv:1906.00524.

[85] M. Carrega, J. Kim, and D. Rosa, Unveiling operator growth using spin correlation functions, Entropy 23, 587 (2021)

[86] D. J. Luitz and Y. Bar Lev, Information propagation in isolated quantum systems, Phys. Rev. B 96, 020406(R) (2017).

[87] C. B. Dağ, L.-M. Duan, and K. Sun, Topologically induced prescrambling and dynamical detection of topological phase transitions at infinite temperature, Phys. Rev. B 101, 104415 (2020). 
[88] T. Gorin, T. Prosen, T. H. Seligman, and M. Žnidarič, Dynamics of Loschmidt echoes and fidelity decay, Phys. Rep. 435, 33 (2006).

[89] B. Yan, L. Cincio, and W. H. Zurek, Information Scrambling and Loschmidt Echo, Phys. Rev. Lett. 124, 160603 (2020).
[90] Z. Liu, A. Wozniakowski, and A. M. Jaffe, Quon 3D language for quantum information, Proc. Natl. Acad. Sci. U.S.A. 114, 2497 (2017).

[91] B. Collins and P. Śniady, Integration with respect to the Haar measure on unitary, orthogonal and symplectic group, Commun. Math. Phys. 264, 773 (2006). 